\title{
A Topological Approach to Full Belief
}

\author{
Alexandru Baltag ${ }^{1} \cdot$ Nick Bezhanishvili ${ }^{1}$. \\ Aybüke Özgün1 ${ }^{1}$ (D) Sonja Smets ${ }^{1}$
}

Received: 7 December 2015 / Accepted: 4 May 2018 / Published online: 22 May 2018

(C) The Author(s) 2018

\begin{abstract}
Stalnaker (Philosophical Studies, 128(1), 169-199 2006) introduced a combined epistemic-doxastic logic that can formally express a strong concept of belief, a concept of belief as 'subjective certainty'. In this paper, we provide a topological semantics for belief, in particular, for Stalnaker's notion of belief defined as 'epistemic possibility of knowledge', in terms of the closure of the interior operator on extremally disconnected spaces. This semantics extends the standard topological interpretation of knowledge (as the interior operator) with a new topological semantics for belief. We prove that the belief logic KD45 is sound and complete with respect to the class of extremally disconnected spaces and we compare our approach to a different topological setting in which belief is interpreted in terms of the derived set operator. We also study (static) belief revision as well as belief dynamics by providing a topological semantics for conditional belief and belief update modalities, respectively. Our setting based on extremally disconnected spaces, however, encounters problems when extended with dynamic updates. We then propose a solution consisting in interpreting belief in a similar way based on hereditarily extremally disconnected spaces, and axiomatize the belief logic of hereditarily extremally disconnected spaces. Finally, we provide a complete axiomatization of
\end{abstract}

Aybüke Özgün

a.ozgun@uva.nl

Alexandru Baltag

thealexandrubaltag@gmail.com

Nick Bezhanishvili

n.bezhanishvili@uva.nl

Sonja Smets

s.j.1.smets@uva.nl

1 ILLC, University of Amsterdam, Amsterdam, Netherlands 
the logic of conditional belief and knowledge, as well as a complete axiomatization of the corresponding dynamic logic.

Keywords Epistemic logic - Doxastic logic - Topological semantics · (Hereditarily) Extremally disconnected spaces · Conditional beliefs · Updates · Completeness · Axiomatization

\section{Introduction}

Edmund Gettier's famous counterexamples against the justified true belief (JTB) account of knowledge [32] invited an interesting and extensive discussion among formal epistemologists and philosophers concerned with understanding the correct relation between knowledge and belief, and, in particular, with identifying the exact properties and conditions that distinguishes a piece of belief from a piece of knowledge and vice versa. Various proposals in the literature analysing the knowledge-belief relation can be classified in two categories: (1) the ones that start with the weakest notion of true justified (or justifiable) belief and add conditions in order to argue that they establish a "good" (e.g. factive, correctly-justified, unrevisable, coherent, stable, truth-sensitive) notion of knowledge by enhancing the conditions in the JTB analysis of knowledge; and (2) the ones that take knowledge as the primitive concept and start from a chosen notion of knowledge and weaken it to obtain a "good" (e.g. consistent, introspective, possibly false) notion of belief. Most research in formal epistemology follows the first approach. In particular, the standard topological semantics for knowledge (in terms of the interior operator) can be included within this first approach, as based on a notion of knowledge as "correctly justified belief": according to the interior semantics, a proposition (set of possible worlds) $P$ is known at the real world $x$ if there exists some "true evidence" (i.e. an open set $U$ containing the real world $x$ ) that entails $P$ (i.e. $U \subseteq P$ ). Other responses to the Gettier challenge falling under this category include, among others, the defeasibility analysis of knowledge [35, 39], the sensitivity account [45], the contextualist account [24] and the safety account [51]. ${ }^{1}$

While most research in formal epistemology follows the first approach, the second approach has to date received much less attention from formal logicians. This is rather surprising, since such a "knowledge-first" approach, which challenges "conceptual priorty of belief over knowledge", has been persuasively defended by one of the most influential contemporary epistemologists (Williamson [69]). The only formal account following this second approach that we are aware of (prior to our own work) is the one given by Stalnaker [53], using a relational semantics for knowledge, based on Kripke models in which the accessibility relation is a directed preorder. In this

\footnotetext{
${ }^{1}$ For an overview of responses to the Gettier challenge and a detailed discussion, we refer the reader to $[33,49]$.
} 
setting, Stalnaker argues that the "true" logic of knowledge is the modal logic $\mathbf{S 4 . 2}$ and that belief can be defined as the epistemic possibility of knowledge. ${ }^{2}$ In other words, believing $p$ is equivalent to "not knowing that you don't know" $p$ :

$$
B p=\neg K \neg K p .
$$

Stalnaker justifies this identity from first principles based on a particular notion of belief, namely belief as "subjective certainty". Stalnaker refers to this concept as "strong belief", but we prefer to call it full belief. ${ }^{3}$ What is important about this type of belief is that it is subjectively indistinguishable from knowledge: an agent "fully believes" $p$ iff she "believes that she knows" $p$.

Indeed, Stalnaker proceeds to formalize AGM belief revision [3], based on a special case of the above semantics, in which the accessibility relation is assumed to be a weakly connected preorder, and (conditional) beliefs are defined by minimization. This validates the AGM principles for belief revision.

In this paper we generalize Stalnaker's formalization, making it independent from the concept of plausibility order and from relational semantics, to a topological setting (motivation for topological semantics for epistemic logic is presented in Section 2.3). In fact, when we interpret knowledge and belief as set-theoretical operators, Stalnaker's axioms naturally lead to a topological semantics that extends the standard topological interpretation of knowledge as interior operator with a new topological semantics for belief, given by the closure of the interior operator with respect to an extremally disconnected topology (see Theorem 4). We compare our new semantics with the older topological interpretation of belief in terms of Cantor derivative, giving several arguments in favour of our semantics. We prove that the logic of knowledge and belief with respect to our semantics is completely axiomatized by Stalnaker's epistemic-doxastic principles. Furthermore, we show that the complete logic of knowledge in this setting is the system S4.2, while the complete logic of belief on extremally disconnected spaces is the standard system KD45.

We moreover focus on a topological semantics for belief revision, assuming the distinction between static and dynamic conditioning made in, e.g., [7, 8, 58]. We examine the corresponding "static" conditioning, by giving a topological semantics for conditional belief $B^{\varphi} \psi$. We formalize a notion of conditional belief $B^{\varphi} \psi$ by relativizing the semantic clause for a simple belief modality to the extension of the learnt formula $\varphi$ and first give a complete axiomatization of the logic of knowledge and conditional beliefs based on extremally disconnected spaces. This topological interpretation of conditional belief also allows us to model static belief revision of a more general type than axiomatized by the AGM theory: the topological model val-

\footnotetext{
${ }^{2}$ Note that Stalnaker considers in his work [53] also several other variations such as $\mathbf{S 4 . 4 , ~ S 4 F , ~ S 5 . ~}$

${ }^{3}$ We adopt this terminology both because we want to avoid the clash with the very different notion of strong belief (due to Battigalli and Siniscalchi [12]) that is standard in epistemic game theory, and because we think that the intuitions behind Stalnaker's notion are very similar to the ones behind Van Fraassen's probabilistic concept of full belief [65].
} 
idates the (appropriate versions of) AGM axioms 1-7, but not necessarily the axiom 8 , though it does validate a weaker version of this axiom. ${ }^{4}$

The above setting, however, comes with a problem when extended to a dynamic one by adding update modalities in order to capture the action of learning (conditioning with) new "hard" (true) information $P .5$ In general, conditioning with new "hard" (true) information $P$ is modeled by simply deleting the "non- $P$ " worlds from the initial model. Its natural topological analogue, as recognized in $[10,11$, 71] among others, is a topological update operator, using the restriction of the original topology to the subspace induced by the set $P$. This interpretation, however, cannot be implemented smoothly on extremally disconnected spaces due to their nonhereditary nature: we cannot guarantee that the subspace induced by any arbitrary true proposition $P$ is extremally disconnected since extremally disconnectedness is not a hereditary property and thus the structural properties, in particular extremally disconnectedness, of our topological models might not be preserved. We proposed a different solution for this problem in [5] via arbitrary topological spaces. In particular, [5] introduces a different topological semantics for belief based on all topological spaces in terms of the interior of the closure of the interior operator and models updates on arbitrary topological spaces. In this paper, however, we propose another solution for this problem via hereditarily extremally disconnected spaces. Hereditarily extremally disconnected spaces are those whose subspaces are still extremally disconnected. By restricting our attention to this class of spaces, we guarantee that any model restriction preserves the important structural properties that make the axioms of the corresponding system sound, in this case, extremally disconnectedness of the initial model. We then interpret updates $\langle! \varphi\rangle \psi$ again as a topological update operator using the restriction of the initial topology to its subspace induced by the new information $\varphi$ and show that we no longer encounter the problem with updates that rises in the case of extremally disconnected spaces: hereditarily extremally disconnected spaces admit updates. Further, we show that while the complete logic of knowledge on hereditarily extremally disconnected spaces is actually $\mathbf{S 4 . 3}$, the complete logic of belief is still KD45. We moreover give a complete axiomatization of the logic of knowledge and conditional beliefs with respect to the class of hereditarily extremally disconnected spaces, as well as a complete axiomatization of the corresponding dynamic logic. In addition, we show that hereditarily disconnected spaces validate the AGM axiom 8, and that therefore our proposed semantics for knowledge and conditional beliefs captures the AGM theory as a theory of static belief revision.

Our work in the current paper has also led to an alternative approach to topological semantics for justified belief and knowledge presented in [6], which takes a more coherentist view on evidence as a source of a rational agent's justified belief and knowledge.

\footnotetext{
${ }^{4} \mathrm{AGM}$ theory is considered to be static in the sense that it captures "the agent's changing beliefs about an unchanging world" [8, p. 14] This static interpretation of AGM theory is mimicked by conditional beliefs in a modal framework, in the style of dynamic epistemic logic, and embedded in the complete system Conditional Doxastic Logic (CDL) introduced by Baltag and Smets in [7, 8].

${ }^{5}$ This term is used to denote information that comes with an inherent warranty of veracity, e.g. because of originating from an infallibly truthful source (see, e.g., [59]).
} 
This work can be seen as an extension of [4]: while the results in [4] and Section 3 of the current paper coincide, the proofs of our important results can only be found in the latter. The soundness and completeness results presented in [4] are merely based on extremally disconnected spaces. In this paper, however, we go further. We model knowledge and belief on hereditarily extremally disconnected spaces and propose a topological semantics for conditional beliefs and updates based on these spaces. ${ }^{6}$

The paper is organized as follows. In Section 2 we provide the topological preliminaries used throughout this paper (Section 2.1) and the interior-based topological semantics for knowledge as well as the topological soundness and completeness

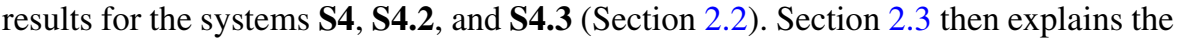
motivation behind the use of the interior operator as a knowledge modality, where the main focus will be on the underlying evidence-based interpretation. Section 3 introduces Stalnaker's combined logic and briefly outlines his analysis regarding the relation between knowledge and belief. We then propose a topological semantics for the system, in particular a topological semantics for full belief. We continue with investigating the unimodal fragments S4.2 for knowledge and KD45 for belief of Stalnaker's system, and give topological completeness results for these logics, again with respect to the class of extremally disconnected spaces. We also compare our topological belief semantics with Steinsvold's co-derived set semantics [54] and argue in favor of ours in this section. Section 4 focuses on a topological semantics for belief revision, assuming the distinction between static and dynamic belief revision and presents the semantics for conditional beliefs and updates, respectively. Finally we conclude with Section 5 by giving a brief summary of this work and pointing out a number of directions for future research.

Throughout the paper we assume familiarity with basic modal logic, Kripke semantics, and axiomatizations of standard epistemic and doxastic logics such as S4, S4.2, S4.3, and KD45. We refer the reader who is not familiar with the aforementioned topics to [19, Chapters 1 and 4].

\section{Background}

\subsection{Topological Preliminaries}

We start by introducing the basic topological concepts that will be used throughout this paper. For a more detailed discussion of general topology we refer the reader to $[25,26]$.

A topological space is a pair $(X, \tau)$, where $X$ is a non-empty set and $\tau$ is a family of subsets of $X$ containing $X$ and $\emptyset$, and is closed under finite intersections and arbitrary unions. The set $X$ is called a space. The subsets of $X$ belonging to $\tau$ are called open sets (or opens) in the space; the family $\tau$ of open subsets of $X$ is also called a topology on $X$. Complements of opens are called closed sets. An open set

\footnotetext{
${ }^{6}$ Chapter 4 of Aybüke Özgün's Ph.D. dissertation [47] was developed based on an earlier version of this paper titled as 'The topological theory of belief'.
} 
Table 1 Kuratowski axioms
(I1) $\operatorname{Int}(X)=X$
(C1) $\mathrm{Cl}(\emptyset)=\emptyset$
(I2) $\operatorname{Int}(A) \subseteq A$
(C2) $A \subseteq \mathrm{Cl}(A)$
(I3) $\operatorname{Int}(A \cap B)=\operatorname{Int}(A) \cap \operatorname{Int}(B)$
(C3) $\mathrm{Cl}(A \cup B)=\mathrm{Cl}(A) \cup \mathrm{Cl}(B)$
(I4) $\operatorname{Int}(\operatorname{Int}(A))=\operatorname{Int}(A)$
(C4) $\mathrm{Cl}(\mathrm{Cl}(A))=\mathrm{Cl}(A)$

containing $x \in X$ is called an open neighbourhood of $x$. The interior $\operatorname{Int}(A)$ of a set $A \subseteq X$ is the largest open set contained in $A$ whereas the closure $\mathrm{Cl}(A)$ of $A$ is the least closed set containing $A$. In other words,
- $\operatorname{Int}(A)=\bigcup\{U \in \tau: U \subseteq A\}$
- $\mathrm{Cl}(A)=\bigcap\{F \subseteq X: X \backslash F \in \tau, A \subseteq F\}$

It is easy to see that $\mathrm{Cl}$ is the De Morgan dual of Int (and vice versa) and can be written as $\operatorname{Cl}(A)=X \backslash \operatorname{Int}(X \backslash A)$. It is well known that the interior Int and the closure $\mathrm{Cl}$ operators of a topological space $(X, \tau)$ satisfy the following properties (the so-called Kuratowski axioms) for any $A, B \subseteq X$ (see Table 1 ): ${ }^{7}$

A point $x \in X$ is called a limit point (or accumulation point) of a set $A \subseteq X$ if for each open neighborhood $U$ of $x$, we have $A \cap(U \backslash\{x\}) \neq \emptyset$. The set of all limit points of $A$ is called the derived set of $A$ and is denoted by $d(A)$. For any $A \subseteq X$, we also let $t(A)=X \backslash d(X \backslash A)$. We call $t(A)$ the co-derived set of $A$. Moreover, a set $A \subseteq X$ is called dense-in-itself if $A \subseteq d(A)$. A space $X$ is called dense-in-itself if $X=d(X)$.

Example For any non-empty set $X,(X, \mathcal{P}(X))$ is a topological space and every set $A \subseteq X$ is both closed and open (i.e., clopen). Another standard example of a topological space is the real line $\mathbb{R}$ with the family $\tau$ of open intervals and their countable unions. If $A=[1,2)$, then $\operatorname{Int}([1,2))=(1,2)$ (the largest open interval included in $[1,2))$ and $\mathrm{Cl}([1,2))=[1,2]$ (the least closed interval containing [1, 2)) (see Fig. 1). Moreover, it is not hard to see that $d([1,2))=[1,2], t([1,2))=(1,2)$, and $(\mathbb{R}, \tau)$ is dense-in-itself.

Definition 1 Given a topological space $(X, \tau)$ and a non-empty set $P \subseteq X$, a space $\left(P, \tau_{P}\right)$ is called a subspace of $(X, \tau)$ where $\tau_{P}=\{U \cap P: U \in \tau\}$.

We can define the closure operator $\mathrm{Cl}_{\tau_{P}}$ and the interior operator $\operatorname{Int}_{\tau_{P}}$ of the subspace $\left(P, \tau_{P}\right)$ in terms of the closure and the interior operators of the space $(X, \tau)$ as follows:
- $\mathrm{Cl}_{\tau_{P}}(A)=\mathrm{Cl}(A) \cap P$
- $\operatorname{Int}_{\tau_{P}}(A)=\operatorname{Int}(P \rightarrow A) \cap P$,

where $P \rightarrow A:=(X \backslash P) \cup A$ is the set-theoretic version of material implication.

\footnotetext{
${ }^{7}$ The properties (I1) - (I4) (and, dually, (C1) - (C4)) are what render the knowledge modality interpreted as the topological interior operator an $\mathbf{S 4}$-type modality. We will elaborate on this in Section 2.2.3.
} 


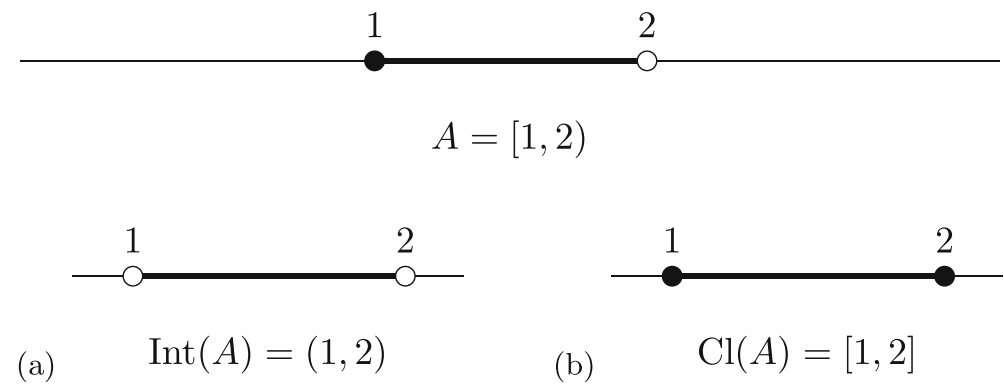

Fig. 1 The natural topology on $\mathbb{R}$

\subsection{The Interior Semantics for Modal Logic}

In this section, we provide the formal background for the interior-based topological semantics for the basic modal logic that originates from the work of McKinsey [41], and McKinsey and Tarski [42] (some of the ideas could already be found in [55] and [57]). In this semantics the modal operator $\square$ is interpreted on topological spaces as the interior operator. These investigations took place in an abstract, mathematical context, independent from epistemic/doxastic considerations. In [42], McKinsey and Tarski not only proved that the modal system $\mathbf{S 4}$ is the logic of all topological spaces (under the above-mentioned interpretation), but also showed that it is the logic of any dense-in-itself separable metric space, such as the rational line $\mathbb{Q}$, the real line $\mathbb{R}$, and the Cantor space, among others. This approach paved the way for a whole new area of spatial logics, establishing a long standing connection between modal logic and topology (see, e.g., [2] for a survey on this topic, in particular, see [60]). Moreover, the completeness results concerning the epistemic system $\mathbf{S 4}$ have naturally attracted epistemic logicians, and led to an epistemic re-evaluation of the interior semantics, seeing topologies as models for information. Borrowing the use of topological models for intuitionistic languages where opens are treated as 'observable properties' or 'pieces of evidence' about where the actual state is, the interior semantics has been considered to provide a deeper insight into the evidential interpretation of knowledge, especially compared to the rather standard and more commonly used relational semantics.

In the following, we briefly introduce the so-called topological interior semantics and explain how and why it constitutes a satisfactory interpretation for (evidencebased) knowledge, and, consequently, why — in certain contexts-it forms a richer semantics than the relational semantics. While stating some important completeness results (concerning logics of knowledge) of previous works, we also explain the connection between the interior semantics and standard Kripke semantics. The presentation of this section follows [60, Section 2]. The reader who is familiar with the source and topic should feel free to continue with Section 3. 


\subsubsection{Syntax and Semantics}

We consider the standard unimodal language $\mathcal{L}_{K}$ with a countable set of propositional letters Prop, Boolean operators $\neg, \wedge$ and a modal operator $K$. Formulas of $\mathcal{L}_{K}$ are defined as usual by the following grammar

$$
\varphi::=p|\neg \varphi| \varphi \wedge \varphi \mid K \varphi
$$

where $p \in$ Prop. Abbreviations for the connectives $\vee, \rightarrow$ and $\leftrightarrow$ are standard. Moreover, the epistemic possibility operator $\langle K\rangle \varphi$ is defined as $\neg K \neg \varphi$ and $\perp:=p \wedge \neg p$.

Given a topological space $(X, \tau)$, we define a topological model, or simply, a topo-model (based on $(X, \tau))$ to be $\mathcal{M}=(X, \tau, \nu)$ where $X$ and $\tau$ are as before and $v:$ Prop $\rightarrow \mathcal{P}(X)$ is a valuation function.

Definition 2 Given a topo-model $\mathcal{M}=(X, \tau, v)$ and a state $x \in X$, the interior semantics for the language $\mathcal{L}_{K}$ is defined recursively as follows:

$$
\begin{array}{ll}
\mathcal{M}, x \models p & \text { iff } \quad x \in v(p) \\
\mathcal{M}, x \models \neg \varphi & \text { iff } \operatorname{not} \mathcal{M}, x \models \varphi \\
\mathcal{M}, x \models \varphi \wedge \psi & \text { iff } \quad \mathcal{M}, x \models \varphi \text { and } \mathcal{M}, x \models \psi \\
\mathcal{M}, x \models K \varphi & \text { iff } \quad(\exists U \in \tau)(x \in U \text { and } \forall y \in U, \mathcal{M}, y \models \varphi)
\end{array}
$$

where $p \in$ Prop.

We let $\llbracket \varphi \rrbracket^{\mathcal{M}}=\{x \in X: \mathcal{M}, x \models \varphi\}$ denote the truth set of a formula $\varphi$ in topo-model $\mathcal{M}$. We omit the superscript for the model when it is clear from the context. It is now easy to see that the semantic clauses for $K$ and $\langle K\rangle$ give us exactly the interior and the closure operators of the corresponding model, respectively. In other words, according to the interior semantics, we have

$$
\begin{aligned}
& \llbracket K \varphi \rrbracket=\operatorname{Int}(\llbracket \varphi \rrbracket) \\
& \llbracket\langle K\rangle \varphi \rrbracket=\operatorname{Cl}(\llbracket \varphi \rrbracket) .
\end{aligned}
$$

We call a formula $\varphi$ true in a topo-model $\mathcal{M}=(X, \tau, v)$, denoted by $\mathcal{M} \models \varphi$, if $\mathcal{M}, x \models \varphi$ for all $x \in X$, and it is valid in a topological space $\mathcal{X}=(X, \tau)$, denoted by $\mathcal{X} \models \varphi$, if $\mathcal{M} \models \varphi$ for every topo-model $\mathcal{M}$ based on $\mathcal{X}$. Moreover, we say $\varphi$ is valid in a class $\mathcal{K}$ of topological spaces, denoted by $\mathcal{K} \models \varphi$, if $\mathcal{X} \models \varphi$ for every member of this class, and it is valid, denoted by $\models \varphi$, if it is valid in the class of all topological spaces. Soundness and completeness with respect to the interior semantics are defined as usual.

\subsubsection{Connection between Kripke Frames and Topological Spaces}

As is well known, there is a tight link between the relational semantics and the interior semantics at the level of reflexive and transitive frames: every reflexive and transitive Kripke frame corresponds to an Alexandroff space. The class of reflexive and transitive frames therefore forms a subclass of all topological spaces. This connection does not only help us to see how the interior semantics and the relational semantics relate to each other and how the former extends the latter, but it also provides a method 
to prove topological completeness results by using the already established results for the relational counterpart.

Let us now fix some notation and terminology. We denote a Kripke frame by $\mathcal{F}=$ $(X, R)$, a Kripke model by $M=(X, R, v)$ and we let $\|\varphi\|^{M}$ denote the truth set of formula $\varphi$ in a Kripke model $M=(X, R, v)$. A topological space $(X, \tau)$ is called Alexandroff if $\tau$ is closed under arbitrary intersections, i.e., $\bigcap \mathcal{A} \in \tau$ for any $\mathcal{A} \subseteq \tau$. Equivalently, a topological space $(X, \tau)$ is Alexandroff iff every point in $X$ has a least open neighborhood. A topo-model $\mathcal{M}=(X, \tau, v)$ is called an Alexandroff model if $(X, \tau)$ is an Alexandroff space.

Given a reflexive and transitive Kripke frame $\mathcal{F}$, we can construct an Alexandroff space $\left(X, \tau_{R}\right)$ by defining $\tau_{R}$ to be the set of all up-sets ${ }^{8}$ of $\mathcal{F}$. The up-set $R(x)=$ $\{y \in X: x R y\}$ forms the smallest open neighborhood containing the point $x$. Conversely, for every topological space $(X, \tau)$, we define a specialization preorder $R_{\tau}$ on $X$ by

$$
x R_{\tau} y \text { iff } x \in \mathrm{Cl}(\{y\}) \text { iff }(\forall U \in \tau)(x \in U \text { implies } y \in U) .
$$

$\left(X, R_{\tau}\right)$ is therefore a reflexive and transitive Kripke frame. Moreover, we have that $R=R_{\tau_{R}}$, and that $\tau=\tau_{R_{\tau}}$ if and only if $(X, \tau)$ is Alexandroff (see, e.g., [60, p. 238]). Hence, there is a natural one-to-one correspondence between reflexive and transitive Kripke models and Alexandroff models. Moreover, the evaluation of modal formulas in a reflexive and transitive Kripke model coincides with their evaluation in the corresponding Alexandroff model, as stated in following proposition (see, e.g., [48, p. 306]):

Proposition 1 For all reflexive and transitive Kripke models $M=(X, R, v)$ and all $\varphi \in \mathcal{L}_{K}$,

where $\mathcal{M}_{\tau_{R}}=\left(X, \tau_{R}, v\right)$.

$$
\|\varphi\|^{M}=\llbracket \varphi \rrbracket^{\mathcal{M}_{\tau_{R}}}
$$

Therefore, reflexive and transitive Kripke models and Alexandroff models are just different representations of each other with respect to the language $\mathcal{L}_{K}$. In particular, the modal equivalence stated in Proposition 1 constitutes the key step that allows us to use the relational completeness results to prove completeness with respect to the interior semantics.

\subsubsection{Topo-Completeness of $\mathbf{S 4 , S 4 . 2}$ and $\mathbf{S 4 . 3}$}

Having explained the connection between reflexive-transitive Kripke models and Alexandroff models, we can now state the topological completeness results for $\mathbf{S 4}$ and its two normal extensions S4.2 and S4.3 that are of particular interests in this paper: our work is built on the interior semantics for knowledge and topological completeness results of the aforementioned logics under this semantics.

Given the interior semantics, it is not hard to see that the Kuratowski axioms of the interior operator are the axioms of the system $\mathbf{S 4}$ written in topological terms (see

\footnotetext{
${ }^{8} \mathrm{~A}$ set $A \subseteq X$ is called an $u$-set of $(X, R)$ if for each $x, y \in X, x R y$ and $x \in A$ imply $y \in A$.
} 
Table 2 S4 vs. Kuratowski axioms

\begin{tabular}{lll}
\hline & S4 axioms and rules & Kuratowski axioms \\
\hline$(\mathrm{K})$ & $K(\varphi \wedge \psi) \leftrightarrow(K \varphi \wedge K \psi)$ & $\operatorname{Int}(A \cap B)=\operatorname{Int}(A) \cap \operatorname{Int}(B)$ \\
$(\mathrm{T})$ & $K \varphi \rightarrow \varphi$ & $\operatorname{Int}(A) \subseteq A$ \\
$(4)$ & $K \varphi \rightarrow K K \varphi$ & $\operatorname{Int}(A) \subseteq \operatorname{Int}(\operatorname{Int}(A))$ \\
$(\mathrm{Nec})$ & from $\phi$, infer $K \varphi$ & $\operatorname{Int}(X)=X$ \\
\hline
\end{tabular}

Table 2). This implies the soundness of $\mathbf{S 4}$ with respect to the class of all topological spaces under the interior semantics (see, e.g., [17, 48, 60]). For completeness, we use the connection between Kripke frames and topological spaces presented in Section 2.2.2. ${ }^{9}$ In fact, Proposition 1 entails the following general result regarding all Kripke complete normal extensions of $\mathbf{S 4}$.

Proposition 2 [60] Every normal extension of $\mathbf{S 4}$ that is complete with respect to the standard Kripke semantics is also complete with respect to the interior semantics.

We can therefore prove completeness of the Kripke complete extensions of $\mathbf{S 4}$ with respect to the interior semantics via their relational completeness. What makes the interior semantics more general than Kripke semantics is tied to soundness. For example, S4 is not only sound with respect to Alexandroff spaces, but also with respect to all topological spaces.

Theorem 1 [42] S4 is sound and complete with respect to the class of all topological spaces under the interior semantics.

Since the normal extensions S4.2 and S4.3 of S4 are of particular interest in our work, we also elaborate on the topological soundness and completeness of these two systems.

$\mathbf{S 4 . 2}$ is a strengthening of $\mathbf{S 4}$ defined as

$$
\mathbf{S 4 . 2}=\mathbf{S 4}+(\langle K\rangle K \varphi \rightarrow K\langle K\rangle \varphi)
$$

where $\mathbf{L}+\varphi$ is the smallest normal modal logic containing $\mathbf{L}$ and $\varphi$. It is well known, see e.g., [19] or [22], that $\mathbf{S 4 . 2}$ is sound and complete with respect to reflexive, transitive and directed Kripke frames. Recall that a Kripke frame $(X, R)$ is called directed $^{10}$ (see Fig. 2) if

$$
(\forall x, y, z)(x R y \wedge x R z) \rightarrow(\exists u)(y R u \wedge z R u) .
$$

The directedness condition on Kripke frames is needed to ensure the validity of (.2)-axiom $\langle K\rangle K \varphi \rightarrow K\langle K\rangle \varphi$, however, in the interior semantics it is a special case of a more general condition called extremally disconnectedness:

\footnotetext{
${ }^{9}$ Alternatively, one can also build a canonical topological model in order to prove the completeness of S4. For the details of this method, see, e.g., [60, Section 2.4.2].

${ }^{10}$ Directedness is also called confluence or the Church-Rosser property.
} 
Fig. 2 Directedness

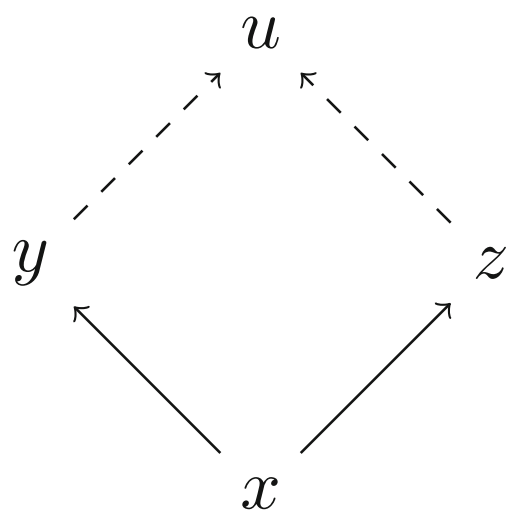

Definition 3 A topological space $(X, \tau)$ is called extremally disconnected if the closure of each open subset of $X$ is open.

For example, Alexandroff spaces corresponding to reflexive, transitive and directed Kripke frames are extremally disconnected. More precisely, for a given reflexive, transitive and directed Kripke frame $(X, R)$, the space $\left(X, \tau_{R}\right)$ is extremally disconnected (see, [46, Proposition 3] for its proof). It is also well known that topological spaces that are Stone-dual to complete Boolean algebras, e.g., the Stone-Čech compactification $\beta(\mathbb{N})$ of the set of natural numbers with a discrete topology, are extremally disconnected [50].

Similarly to the case of the standard Kripke semantics, in the interior semantics extremally disconnectedness is needed in order to ensure the validity of the (.2)axiom. More accurately, (.2)-axiom characterizes extremally disconnected spaces under the interior semantics:

Proposition 3 [29, 60] For all topological spaces $(X, \tau)$,

$\langle K\rangle K \varphi \rightarrow K\langle K\rangle \varphi$ is valid in $(X, \tau)$ iff $(X, \tau)$ is extremally disconnected.

Proposition 3 and topological soundness of S4 imply that $\mathbf{S 4 . 2}$ is sound with respect to the class of extremally disconnected spaces. As reflexive, transitive and directed Kripke frames correspond to extremally disconnected Alexandroff spaces, the following topological completeness result follows from the completeness of $\mathbf{S 4 . 2}$ with respect to the standard Kripke semantics and Proposition 2:

Theorem 2 [29, 60] $\boldsymbol{S 4 . 2}$ is sound and complete with respect to the class of extremally disconnected spaces under the interior semantics. ${ }^{11}$

\footnotetext{
${ }^{11}$ In fact, this result was already known to Leo Esakia in the 1980's - personal communication of the second named author.
} 
Another logic of knowledge that is of particular interest in this paper is the system S4.3. Recall that $\mathbf{S 4 . 3}$ as well is a strengthening of $\mathbf{S 4}$ (and also of $\mathbf{S 4 . 2}$ ) defined as

$$
\mathbf{S 4 . 3}:=\mathbf{S 4}+K(K \varphi \rightarrow \psi) \vee K(K \psi \rightarrow \varphi) .
$$

Kripke semantics for this system is also well studied and it is well known, see e.g., [19], that $\mathbf{S 4 . 3}$ is sound and complete with respect to reflexive, transitive and linear Kripke frames. Recall that a Kripke frame $(X, R)$ is called linear if ${ }^{12}$

$$
(\forall x, y, z)((x R y \wedge x R z) \rightarrow(y R z \vee z R y \vee y=z)) .
$$

The linearity condition on Kripke frames is needed to ensure the validity of (.3)axiom $K(K \varphi \rightarrow \psi) \vee K(K \psi \rightarrow \varphi)$. However, to guarantee the validity of this axiom on topological spaces under the interior semantics, we need the property of hereditarily extremally disconnectedness:

Definition 4 A topological space $(X, \tau)$ is called hereditarily extremally disconnected (h.e.d.) if every subspace of $(X, \tau)$ is extremally disconnected.

For hereditarily extremally disconnected spaces, we can think of Alexandroff spaces corresponding to total preorders, in particular, corresponding to reflexive, transitive and linear Kripke frames. Another interesting and non-Alexandroff example of a hereditarily extremally disconnected space is the topological space $(\mathbb{N}, \tau)$ where $\mathbb{N}$ is the set of natural numbers and $\tau=\{\emptyset$, all cofinite subsets of $\mathbb{N}\}$. Furthermore, every countable Hausdorff extremally disconnected space is hereditarily extremely disconnected [20]. For more examples of hereditarily extremally disconnected spaces, we refer to [20].

Theorem 3 [14] S4.3 is sound and complete with respect to the class of hereditarily extremally disconnected spaces under the interior semantics.

\subsection{The Motivation behind Knowledge as Interior}

Having presented the interior semantics, we can now elaborate on its epistemic significance that has inspired our work in this paper.

\subsubsection{The Interior Semantics is Naturally Epistemic and Extends the Relational Semantics}

The initial reason as to why the topological interior operator can be considered as knowledge is inherent to the properties of this operator. As noted in Section 2.2.3, the Kuratowski axioms (I1)-(I4) correspond exactly to the axioms of the system S4, when $K$ is interpreted as the interior modality (see Table 2 for the one-to-one correspondence). Therefore, elementary topological operators such as the interior

\footnotetext{
${ }^{12}$ This property is also called no branching to the right (see, e.g., [19, p. 195]) and it boils down to $(\forall x, y, z)((x R y \wedge x R z) \rightarrow(y R z \vee z R y))$ when $R$ is reflexive.
} 
operator, or, dually, the closure operator produces the epistemic logic S4 with no need for additional constraints (also see Theorem 1). In other words, in its most general form, topologically modeled knowledge is Factive and Positively Introspective, however, it does not necessarily possess stronger properties. On the other hand, this in no way limits the usage of interior semantics for stronger epistemic systems. In accordance with the case for the relational semantics, we can restrict the class of spaces we work with and interpret stronger epistemic logics such as $\mathbf{S 4 . 2 , ~ S 4 . 3}$ (see Theorems 2 and 3) and S5 in a similar manner (see, e.g., [60, p. 253]). To that end, topological spaces provide sufficiently flexible structures to study knowledge of different strength. They are moreover naturally epistemic since the most general class of spaces, namely the class of all topological spaces, constitutes the class of models of arguably the weakest, yet philosophically the most accepted normal system S4. Moreover, as explained in Sections 2.2.2 and 2.2.3, the relational models for the logic S4, and for its normal extensions, correspond to the proper subclass of Alexandroff models (see Proposition 2). The interior semantics therefore generalizes the standard relational semantics for knowledge.

One may however argue that the above reasons are more of a technical nature showing that the interior semantics works as well as the relational semantics, therefore motivate "why we could use topological spaces" rather than "why we should use topological spaces" to interpret knowledge as opposed to using relational semantics. Certainly the most important argument in favour of the conception of knowledge as the interior operator is of a more 'semantic' nature: the interior semantics provides a deeper insight into the evidence-based interpretation of knowledge.

\subsubsection{Evidence as Open Sets}

The idea of treating 'open sets as pieces of evidence' is adopted from the topological semantics for intuitionistic logic, dating back to the 1930s (see, e.g., [56]). In a topological-epistemological framework, typically, the elements of a given open basis are interpreted as observable evidence, whereas the open sets of the topology are interpreted as properties that can be verified based on the observable evidence. In fact, the connection between evidence and open sets comes to exist at the most elementary level, namely at the level of a subbasis. We can think of a subbasis as a collection of observable evidence that is directly obtained by an agent via, e.g., testimony, measurement, approximation, computation or experiment. The family of directly observable pieces of evidence therefore naturally forms an open topological basis: closure under finite intersection captures an agent's ability to put finitely many pieces into a single piece, i.e., her ability to derive more refined evidence from direct ones by combining finitely many of them together. Therefore, the notion of knowledge as the interior operator yields an evidential interpretation at a purely semantic level. Moreover, the use of opens sets as pieces of evidence intrinsically reflects the fact that the physical measuring devices we use to calculate quantitative valuessuch as the height of a person [18], temperature of the weather, speeding of a car [48], etc.-always come with a certain error range giving an approximation rather than the precise value. Carefully taken measurements with increasingly accurate devices results in better approximations to the actual state, yet being able to know the actual 
state requires taking a measurement with its exact value. This, according to the interior semantics, necessitates having the singleton set of the actual state an open set in the corresponding topology. Elaborating on these points in more technical terms, a proposition $P$ is true at world $x$ if $x \in P$. If an open set $U$ is included in a set $P$, then we can say that proposition $P$ is entailed/supported by evidence $U$ by sharply distinguishing $P$-states from all non- $P$ states. Open neighbourhoods $U$ of the actual world $x$ play the role of sound (correct, truthful) evidence. Therefore, the statement

$$
x \in \operatorname{Int}(P) \text { iff }(\exists U \in \tau)(x \in U \text { and } U \subseteq P)
$$

means that the actual world $x$ is in the interior of $P$ iff there exists a sound piece of evidence $U$ that supports $P$. That is, according to the interior semantics, the agent knows $P$ at $x$ iff she has a sound/correct piece of evidence supporting $P$. So the knowledge-as-interior conception can be seen as an implementation of one of the most widespread intuitive responses to Gettier's challenge: knowledge is “correctly justified belief" (rather than being simply true justified belief). To qualify as knowledge, not only the content of one's belief has to be truthful, but its evidential justification has to be sound. Moreover, open sets will then correspond to properties that are in principle verifiable by the agent: whenever they are true, they are supported by a sound piece of evidence, therefore, can be known. Dually, we have

$$
x \notin \mathrm{Cl}(P) \text { iff }(\exists U \in \tau)(x \in U \text { and } U \subseteq X \backslash P)
$$

meaning that closed sets correspond to falsifiable properties: whenever they are false, they are falsified by a sound piece of evidence. Besides, although it does not play a major role in this paper, we briefly comment on the epistemic role of the set of boundary points. The set of boundary points of a set $P \subseteq X$, denoted by $\operatorname{Bd}(P)$, is defined as $\operatorname{Bd}(P)=\mathrm{Cl}(P) \backslash \operatorname{Int}(P)$. More precisely,

$$
x \in \operatorname{Bd}(P) \text { iff }(\forall U \in \tau)(x \in U \text { implies } U \nsubseteq X \backslash P \text { and } U \nsubseteq P)
$$

Therefore, $x$ is a boundary point of $P$ iff there is no sound piece of evidence that supports neither $P$ nor $\neg P$. In other words, given the aforementioned interpretations of open and closed sets, the set of boundary points corresponds to the properties that are neither verifiable nor falsifiable. These ideas have also been used and developed in $[1,34,66]$ with connections to epistemology, logic, and learning theory.

The interior semantics for knowledge has been extended to multiple agents [61], to common knowledge $[9,63]$ to logics of learning and observational effort $[23,30$, 31,43 ], to topological versions of dynamic-epistemic logic [71] (see [2] for a comprehensive overview on the field). On the other hand, belief on topological spaces, rather surprisingly, has not been investigated and developed as much as knowledge, especially in connection with topological knowledge.

\subsubsection{Belief on Topological Spaces?}

As explained in Section 2.3.2, as far as an evidential interpretation of knowledge is concerned, the interior semantics improves the standard relational semantics, most importantly, for the reason that evidential justification for knowing something is 
embedded in the semantics. It then seems natural to ask whether a topological semantics can also account for notions of (evidentially) justified belief. In the next section, we propose a topological semantics for Stalnaker's notion of belief as subjective certainty [53] in terms of the closure of the interior operator, which, in turn, will answer the above question positive.

One of the crucial properties that distinguishes knowledge from belief is its veracity (formalized by the axiom (T) $K \varphi \rightarrow \varphi$ ). However, no matter how idealized and rational the agent is, it must be possible for her to believe false propositions, yet she is expected to hold consistent beliefs (formalized by the axiom (D) $\neg B \perp$ ). To the best of our knowledge, the first worked out topological semantics for belief is proposed by Steinsvold [54] in terms of the co-derived set operator. According to the co-derived set interpretation of belief,

$$
x \in B P \text { iff }(\exists U \in \tau)(x \in U \text { and } U \backslash\{x\} \subseteq P),
$$

i.e., $x \in B P$ iff $x \in t(P) .{ }^{13}$ Steinsvold [54] was the first to propose to use this semantics to interpret belief, and proved soundness and completeness for the standard belief system KD45. This account still requires having a truthful piece of evidence for the believed proposition, however, the proposition itself does not have to be true. Therefore, it is guaranteed that the agent may hold false beliefs. However, as also discussed in [4, 46], and briefly recapped here, this semantics further guarantees that in any topo-model and any state in this model, there is at least one false belief, that is, the agent always believes the false proposition $X \backslash\{x\}$ at the actual state $x$. This is the case because for any topological space $(X, \tau)$ and $x \in X$, we have $x \notin d(\{x\})$, i.e., $x \in t(X \backslash\{x\})$, therefore, the clause (3) entails that $x \in B(X \backslash\{x\})$ always holds. This is an undesirable and disadvantageous property, especially if we also want to study dynamics such as belief revision, updates or learning. Always believing $X \backslash\{x\}$ prevents the agent to ever learning the actual state unless she believes everything. Formally speaking, $x \in B(\{x\})$ iff the singleton $\{x\}$ is an open, and in this case, the agent believes everything at $x$. In order to avoid these downsides and obtain KD45, we have to work with the so-call DSO-spaces, as shown in [54]. A DSO-space is defined to be a dense-in-itself space (i.e., a space with no singleton opens) in which every derived set $d(A)$ is open.

Moreover, in a setting where knowledge as the interior and belief as the co-derived set operator are studied together, we obtain the equality

$$
K P=P \cap B P,
$$

stating that knowledge is true belief. Therefore, this semantics yields a formalization of knowledge and belief that is subject to well-known Gettier counterexamples [32]. ${ }^{14}$

\footnotetext{
${ }^{13}$ We here note that this topological semantics interpreting the modal operator $\square$ as the co-derived set operator, or dually, $\diamond$ as the derived set operator was also pioneered by McKinsey and Tarski [42], and later extensively developed by the Georgian logic school led and inspired by Esakia, and their collaborators (see, e.g., $[13,15,16,27,28,37])$.

${ }^{14}$ This connection has also been observed in [54, Section 1.11], and an alternative topological semantics for knowledge in terms of clopen sets is suggested without providing any further technical results. Steinsvold [54] does not elaborate on to what extend his proposed semantics for knowledge could give new insight into the Gettier problem and leaves this point open for discussion.
} 
In the next chapter, we propose another topological semantics for belief, in particular, for Stalnaker's notion of belief as subjective certainty [53], in terms of the closure of the interior operator on extremally disconnected spaces.

\section{The Topology of Full Belief and Knowledge}

\subsection{Stalnaker's Combined Logic of Knowledge and Belief}

In his paper [53], Stalnaker focuses on the properties of (justified or justifiable) belief and knowledge and proposes an interesting analysis regarding the relation between the two. As also pointed out in the introduction, most research in the formal epistemology literature concerning the relation between knowledge and belief, in particular, dealing with the attempt to provide a definition of the one in terms of the other, takes belief as a primitive notion and tries to determine additional properties which render a piece of belief knowledge (see, e.g., [24, 35, 39, 45, 49]). In contrast, Stalnaker chooses to start with a notion of knowledge and weakens it to have a "good" notion of belief. He initially considers knowledge to be an S4-type modality and analyzes belief based on the conception of "subjective certainty": from the point of the agent in question, her belief is subjectively indistinguishable from her knowledge.

In his formalization, Stalnaker [53] uses the bimodal language $\mathcal{L}_{K B}$ of knowledge and (full) belief given by the following grammar:

$$
\varphi::=p|\neg \varphi| \varphi \wedge \varphi|K \varphi| B \varphi
$$

where $p \in$ Prop. Abbreviations for the connectives $\vee, \rightarrow$ and $\leftrightarrow$ are standard. The existential modalities $\langle K\rangle \varphi$ and $\langle B\rangle \varphi$ are defined as $\neg K \neg \varphi$ and $\neg B \neg \varphi$ respectively. We will also consider two unimodal fragments $\mathcal{L}_{K}$ (having $K$ as its only modality) and $\mathcal{L}_{B}$ (having only $B$ ) of the language $\mathcal{L}_{K B}$ in later sections.

Table 3 Stalnaker's System KB

\begin{tabular}{lll}
\hline & Stalnaker's Axioms & \\
$(\mathrm{K})$ & $K(\varphi \rightarrow \psi) \rightarrow(K \varphi \rightarrow K \psi)$ & Knowledge is additive \\
$(\mathrm{T})$ & $K \varphi \rightarrow \varphi$ & Knowledge implies truth \\
$(\mathrm{KK})$ & $K \varphi \rightarrow K K \varphi$ & Positive introspection for $K$ \\
$(\mathrm{CB})$ & $B \varphi \rightarrow \neg B \neg \varphi$ & Consistency of belief \\
$(\mathrm{PI})$ & $B \varphi \rightarrow K B \varphi$ & (Strong) positive introspection of $B$ \\
$(\mathrm{NI})$ & $\neg B \varphi \rightarrow K \neg B \varphi$ & (Strong) negative introspection of $B$ \\
$(\mathrm{~KB})$ & $K \varphi \rightarrow B \varphi$ & Knowledge implies Belief \\
$(\mathrm{FB})$ & $B \varphi \rightarrow B K \varphi$ & Full Belief \\
& Inference Rules & \\
$(\mathrm{MP})$ & from $\varphi$ and $\varphi \rightarrow \psi$, infer $\psi$ & Modus Ponens \\
$(\mathrm{Nec})$ & from $\varphi$, infer $K \varphi$ & Necessitation \\
\hline
\end{tabular}


We call Stalnaker's epistemic-doxastic system, given in Table 3, KB. The axioms seem very natural and uncontroversial: ${ }^{15}$ the first three are the $\mathbf{S 4}$ axioms for knowledge; (CB) captures the consistency of beliefs, and in the context of the other axioms will be equivalent to the modal axiom (D) for beliefs: $\neg B \perp$; (PI) and (NI) capture strong versions of introspection of beliefs: the agent knows what she believes and what not; (KB) means that agents believe what they know; and finally, (FB) captures the essence of "full belief" as subjective certainty (the agent believes that she knows all the things that she believes). Finally, the rules of Modus Ponens and Necessitation seem uncontroversial (for implicit knowledge, if not for explicit knowledge) and are accepted by a majority of authors (and in particular, they are implicitly used by Stalnaker). The system KB yields the belief logic KD45 as shown in Proposition 4.

Proposition 4 [53] All axioms of the standard belief system KD45 are provable in the system $\mathbf{K B}$. More precisely, the following axioms are provable in $\mathbf{K B}$ :
(K) $B(\varphi \rightarrow \psi) \rightarrow(B \varphi \rightarrow B \psi)$
(4) $B \varphi \rightarrow B B \varphi$
(D) $B \varphi \rightarrow \neg B \neg \varphi$
(5) $\neg B \varphi \rightarrow B \neg B \varphi$

Proposition 5 [53] The following equivalence is provable in the system $\mathbf{K B}$ :

$$
B \varphi \leftrightarrow\langle K\rangle K \varphi
$$

Proposition 5 constitutes one of the most important features of Stalnaker's combined system KB. ${ }^{16}$ This equivalence allows us to have a combined logic of knowledge and belief in which the only modality is $K$ and the belief modality $B$ is defined in terms of the former. We therefore obtain "...a more economical formulation of the combined belief-knowledge logic..." [53, p. 179]. Moreover, substituting $\langle K\rangle K$ for $B$ in the axiom (CB) results in the modal (.2)-axiom

$$
\langle K\rangle K \varphi \rightarrow K\langle K\rangle \varphi .
$$

Recall that we obtain the logic of knowledge $\mathbf{S 4 . 2}$ by adding the (.2)-axiom to the system $\mathbf{S 4}$ (see Section 2.2.3). If we substitute $\langle K\rangle K$ for $B$ in all the other axioms of KB, they turn out to be theorems of S4.2 [53]. Therefore, given the equivalence $B \varphi \leftrightarrow\langle K\rangle K \varphi$, we can obtain the unimodal logic of knowledge $\mathbf{S 4 . 2}$ by substituting $\langle K\rangle K$ for $B$ in all the axioms of $\mathbf{K B}$ implying that the logic $\mathbf{S 4 . 2}$ by itself forms a unimodal combined logic of knowledge and belief. Stalnaker then argues that his analysis of the relation between knowledge and belief suggests that the "true" logic of knowledge should be $\mathbf{S 4 . 2}$ and that belief can be defined as the epistemic possibility of knowledge:

$$
B \varphi:=\langle K\rangle K \varphi .
$$

This equation leads to our proposal for a topological semantics of (full) belief.

\footnotetext{
${ }^{15}$ What justifies the properties of knowledge and belief stated in $\mathbf{K B}$ may be debatable, though not in the scope of this paper. We refer to [18] for a topological-based reformulation of Stalnaker's system.

${ }^{16}$ Interested reader can find the proof of Proposition 5 in [46, Chapter 4].
} 


\subsection{Our Topological Semantics for Full Belief}

In this section, we introduce a new topological semantics for the language $\mathcal{L}_{K B}$, which is an extension of the interior semantics for knowledge with a new topological semantics for belief given by the closure of the interior operator.

Definition 5 (Topological Semantics for Full Belief and Knowledge) Given a topomodel $\mathcal{M}=(X, \tau, v)$, the semantics for the formulas in $\mathcal{L}_{K B}$ is defined for Boolean cases and $K \varphi$ the same way as in the interior semantics. The semantics for $B \varphi$ is defined as

$$
\llbracket B \varphi \rrbracket^{\mathcal{M}}=\operatorname{Cl}\left(\operatorname{Int}\left(\llbracket \varphi \rrbracket^{\mathcal{M}}\right)\right) .
$$

Truth and validity of a formula is defined the same way as in the interior semantics.

Proposition 6 A topological space validates all the axioms and rules of Stalnaker's system $\boldsymbol{K B}$ (under the semantics given above) iff it is extremally disconnected.

Proof See Appendix A.1.

Having a look at the system KB from a more general semantic perspective, we can argue that the epistemic-doxatic logic $\mathbf{K B}$ naturally induces a topological semantics that is based on extremally disconnected spaces. To explicate, consider the semantic structure $\mathcal{M}=(X, \mathrm{~K}, \mathrm{~B}, v)$, where $X$ is a set of possible worlds and $\mathrm{K}: \mathcal{P}(X) \rightarrow \mathcal{P}(X)$ and $\mathrm{B}: \mathcal{P}(X) \rightarrow \mathcal{P}(X)$ are unary operations on (sub)sets of worlds with no pre-imposed restrictions (in particular, they are not assumed to be topological operators), and $v$ : Prop $\rightarrow \mathcal{P}(X)$ is a standardly defined valuation function. The semantics for the formulas in $\mathcal{L}_{K B}$ on $(X, \mathrm{~K}, \mathrm{~B}, v)$ is defined for Boolean cases in the usual way (see, e.g., Definition 2), whereas the semantic clauses for the modal operators $K$ and $B$ are given by means of the functions $\mathrm{K}$ and $\mathrm{B}$, respectively, as

$$
\begin{aligned}
\llbracket K \varphi \rrbracket^{\mathcal{M}} & =\mathrm{K} \llbracket \varphi \rrbracket^{\mathcal{M}} \\
\llbracket B \varphi \rrbracket^{\mathcal{M}} & =\mathrm{B} \llbracket \varphi \rrbracket^{\mathcal{M}} .
\end{aligned}
$$

As usual, a formula $\varphi \in \mathcal{L}_{K B}$ is valid in $(X, \mathrm{~K}, \mathrm{~B})$ if $\llbracket \varphi \rrbracket^{\mathcal{M}}=X$ for all models $\mathcal{M}=(X, \mathrm{~K}, \mathrm{~B}, v)$ based on $(X, \mathrm{~K}, \mathrm{~B})$.

This way, we have defined an alternative and, in a sense, more general semantics for the language $\mathcal{L}_{K B}$ which interprets $K$ and $B$ via set theoretical operators $\mathrm{K}$ and $\mathrm{B}$, respectively, independently from topologies. However, it is not hard to see that a special case $\left(X, \mathrm{~K}_{\tau}, \mathrm{B}_{\tau}\right)$ of the above semantics for the language $\mathcal{L}_{K B}$ is our proposed topological semantics, where $(X, \tau)$ is a topological space, $\mathrm{K}_{\tau}=$ Int is the interior operator, and $\mathrm{B}_{\tau}=\mathrm{Cl}$ (Int) is the closure of the interior operator with respect to the topology $\tau$. More importantly, a structure $(X, \mathrm{~K}, \mathrm{~B})$ validating all the axioms and rules of $\mathbf{K B}$ is indeed a topological one based on an extremally disconnected space:

Theorem 4 (Topological Representation Theorem) A semantic structure (X, K, B) validates all the axioms and rules of Stalnaker's system $\mathbf{K B}$ iff it is a topological one 
given by an extremally disconnected topology $\tau$ on $X$, such that $\mathrm{K}=\mathrm{K}_{\tau}=$ Int and $\mathrm{B}=\mathrm{B}_{\tau}=\mathrm{Cl}(\mathrm{Int})$.

\section{Proof See Appendix A.2.}

Theorem 4 shows that Stalnaker's axioms form an alternative axiomatization of extremally disconnected spaces, in which both the interior and the closure of the interior are taken to be primitive operators (corresponding to the primitive modalities $K$ and $B$ in $\mathcal{L}_{K B}$, respectively). The conclusion is that our topological semantics is indeed the most general semantics validating Stalnaker's axioms in the sense described above.

Theorem 5 The sound and complete logic of knowledge and belief on extremally disconnected spaces is given by Stalnaker's system $\mathbf{K B}$.

Proof See Appendix A.3.

\subsection{Unimodal Case: the Belief Logic KD45}

As emphasized in the beginning of Section 3, Stalnaker's logic KB yields the system S4.2 as the logic of knowledge and KD45 as the logic of belief (Proposition 4). It has already been proven that $\mathbf{S 4 . 2}$ is sound and complete with respect to the class of extremally disconnected spaces under the interior semantics (Theorem 2). In this section, we investigate the case for KD45 under our proposed semantics for belief. More precisely, we focus on the unimodal case for belief and consider the topological semantics for the unimodal language $\mathcal{L}_{B}$ in which we interpret belief as the closure of interior operator. We name our proposed semantics in this section topological belief semantics. We then prove topological soundness and completeness results for KD45 under the aforementioned semantics. Let us first recall the basic doxastic language $\mathcal{L}_{B}$, the system KD45 and the topological belief semantics for the language $\mathcal{L}_{B}$.

The language $\mathcal{L}_{B}$ is given by

$$
\varphi:=p|\neg \varphi| \varphi \wedge \varphi \mid B \varphi
$$

and we again denote $\neg B \neg$ by $\langle B\rangle$. Recall that

$$
\text { KD45 }=\mathbf{K}+(B \varphi \rightarrow\langle B\rangle \varphi)+(B \varphi \rightarrow B B \varphi)+(\langle B\rangle \varphi \rightarrow B\langle B\rangle \varphi)
$$

and given a topo-model $\mathcal{M}=(X, \tau, v)$, the semantic clauses for the propositional variables and the Boolean connectives are the same as in the interior semantics. For the modal operator $B$, we put

$$
\llbracket B \varphi \rrbracket^{\mathcal{M}}=\mathrm{Cl}\left(\operatorname{Int}\left(\llbracket \varphi \rrbracket^{\mathcal{M}}\right)\right)
$$

and the semantic clause for $\langle B\rangle$ is easily obtained as

$$
\llbracket\langle B\rangle \varphi \rrbracket^{\mathcal{M}}=\operatorname{Int}\left(\mathrm{Cl}\left(\llbracket \varphi \rrbracket^{\mathcal{M}}\right)\right) .
$$

We are now ready to state the main results of this section. 
Theorem 6 The belieflogic KD45 is sound with respect to the class of extremally disconnected spaces under the topological belief semantics. In fact, a topological space $(X, \tau)$ validates all the axioms and rules of the system $\mathrm{KD} 45$ (under the topological belief semantics) iff $(X, \tau)$ is extremally disconnected.

\section{Proof See Appendix A.4.}

Theorem 7 In the topological belief semantics, KD45 is the complete logic of belief with respect to the class of extremally disconnected spaces.

Theorem 7 therefore shows that the logic of extremally disconnected spaces is KD45 when $B$ is interpreted as the closure of the interior operator. The interested reader can find all the details of both soundness and completeness proofs in Appendix A.4 and A.5, respectively. The proof details however can be skipped without loss of continuity. Besides these technical results, the closure-interior semantics of belief comes with an intrinsic philosophical and intuitive value, and certain advantages compared to the co-derived set semantics as elaborated in the next section.

\subsubsection{What Motivates Topological Full Belief}

The closure-interior semantics provides an intuitive interpretation of Stalnaker's conception of (full) belief as subjective certainty. It does so through the definitions of the interior and closure operators and the concepts they represent, namely, the notions of evidence and closeness. We have discussed the role of open sets as pieces of evidence, and of open neighbourhoods of the actual state as pieces of truthful evidence in Section 2.3. Moreover, it is well known that the closure operator captures a topological, qualitative notion of closeness: $x$ is said to be close to a set $A \subseteq X$ iff $x \in \mathrm{Cl}(A)$. Recalling the proposed topological semantics for full belief, given a topological space $(X, \tau)$ and $P \subseteq X$, we have

$$
\begin{aligned}
x \in B P & \text { iff } x \in \mathrm{Cl}(\operatorname{Int}(P)) \\
& \text { iff } x \in \mathrm{Cl}(K(P)) \\
& \text { iff }(\forall U \in \tau)(x \in U \text { implies } U \cap K P \neq \emptyset)
\end{aligned}
$$

Therefore, following (5), topologically, the set of states in which the agent believes $P$ is very close to the set of states in which the agent knows $P$. Taking open sets as evidence pieces, (6) moreover states that an agent (fully) believes $P$ at a state $x$ iff every sound piece of evidence she has at $x$ is consistent with her knowing $P$, i.e., she does not have any truthful evidence that distinguishes the states in which she has belief of $P$ from the states in which she has knowledge of $P$. Belief, under this semantics, therefore becomes subjectively indistinguishable from knowledge. Hence, the closure-interior semantics naturally captures the conception of belief as "subjective certainty".

Moreover, the closure-interior belief semantics improves on the co-derived set semantics for the following reasons: (1) belief as the closure of the interior operator 
does not face the Gettier problem, at least not in the easy way in which the co-derived set semantics does, when considered together with the conception of knowledge as interior. More precisely, knowledge as interior cannot be defined as (justified) true full belief since, in general, $\operatorname{Int}(P) \neq \mathrm{Cl}(\operatorname{Int}(P)) \cap P$, i.e., $K P \neq B P \wedge P$; (2) the class of DSO-spaces with respect to which KD45 is sound and complete under the co-derived set semantics is a proper subclass of the class of extremally disconnected spaces (see Proposition 7), which shows that the closure-interior semantics for KD45 is defined on a larger class of spaces.

Proposition 7 Every DSO-space is extremally disconnected. However, not every extremally disconnected space is a DSO-space.

Proof See Appendix A.6.

In the next section, we study a topological analogue of static conditioningcapturing static belief revision-by providing a topological semantics for conditional beliefs based on extremally disconnected spaces. We then extend the setting for dynamic conditioning by providing semantics for updates.

\section{Topological Models for Belief Revision: Static and Dynamic Conditioning}

Conditioning (with respect to some qualitative plausibility order or to a probability measure) is the most widespread way to model the learning of "hard" information. The prior plausibility/probability assignment (encoding the agent's original beliefs before the learning) is changed to a new such assignment, obtained from the first one by conditioning with the new information $P$. In the qualitative case, this means just restricting the original order to $P$-worlds; while in the probabilistic case, restriction has to be followed by re-normalization (to ensure that the probabilities newly assigned to the remaining worlds add up to 1). In Dynamic Epistemic Logic (DEL), one makes also a distinction between simple ("static") conditioning and dynamic conditioning (also known as "update"). The first essentially corresponds to conditional beliefs: the change is made only locally, affecting only one occurrence of the belief operator $B \varphi$ (which is thus locally replaced by conditional belief $B^{P} \varphi$ ) or of the probability measure (which is locally replaced by conditional probability). In contrast, an update is a global change, at the level of the whole model (thus recursively affecting the meaning of all occurrences of the belief/probability operators). In this section, we investigate the natural topological analogues of static and dynamic conditioning.

\subsection{Static Conditioning: Conditional Beliefs}

In DEL, static belief revision captures the agent's revised beliefs about how the world was before learning new information and is implemented by conditional 
belief operators $B^{\varphi} \psi$. Using van Benthem's terminology, "[c]onditional beliefs preencode beliefs that we would have if we learnt certain things." [58, p. 139]. The statement $B^{\varphi} \psi$ says that "if the agent would learn $\varphi$, then she would come to believe that $\psi$ was the case before the learning" [8, p. 12]. That means conditional beliefs are hypothetical by nature, hinting at possible future belief changes of the agent. In the DEL literature, the semantics for conditional beliefs is generally given in terms of plausibility models (or equivalently, in terms of sphere models), see, e.g., $[8,58,62]$.

In this section, we explore the topological analogue of static conditioning by providing a topological semantics for conditional belief modalities. As conditional beliefs capture hypothetical belief changes of an agent in case she would learn a piece of new information $\varphi$, we can obtain the semantics for a conditional belief modality $B^{\varphi} \psi$ in a natural and standard way by relativizing the semantics for the simple belief modality to the extension of the learnt formula $\varphi$. By relativization we mean a local change in the sense that it only affects one occurrence of the belief modality $B \varphi$. It does not cause a change in the model, i.e. it does not lead to a global change, due to its static nature.

Semantics of Conditional Beliefs To recap, given a topo-model $\mathcal{M}=(X, \tau, v)$ based on an extremally disconnected topology $\tau$, we can describe the extension of a belief formula in the following equivalent ways

$$
\llbracket B \varphi \rrbracket \stackrel{(1)}{=} \mathrm{Cl}(\operatorname{Int}(\llbracket \varphi \rrbracket)) \stackrel{(2)}{=} \operatorname{Int}(\mathrm{Cl}(\operatorname{Int}(\llbracket \varphi \rrbracket))) .
$$

However, when we generalize the belief operator $B$ by relativizing the closure and the interior operators to the extension of a learnt formula $\varphi$ in order to obtain a semantics for conditional belief modalities, the resulting clauses no longer remain equivalent. While the relativization of (1) leads to

$$
\llbracket B^{\varphi} \psi \rrbracket=\mathrm{Cl}(\llbracket \varphi \rrbracket \cap \operatorname{Int}(\llbracket \varphi \rrbracket \rightarrow \llbracket \psi \rrbracket)),
$$

called the basic topological semantics for conditional beliefs, the relativization of (2) results in

$$
\llbracket B^{\varphi} \psi \rrbracket=\operatorname{Int}(\llbracket \varphi \rrbracket \rightarrow \operatorname{Cl}(\llbracket \varphi \rrbracket \cap \operatorname{Int}(\llbracket \varphi \rrbracket \rightarrow \llbracket \psi \rrbracket))),
$$

called the refined topological semantics for conditional beliefs, where $\llbracket \varphi \rrbracket \rightarrow \llbracket \psi \rrbracket$ is used as an abbreviation for $(X \backslash \llbracket \varphi \rrbracket) \cup \llbracket \psi \rrbracket$.

However, for the reasons explained below, the basic semantics (7) does not work well as a generalization of belief on extremally disconnected spaces (and the same arguments hold for the same formalization on hereditarily extremally disconnected spaces). First of all, it validates the equivalence

$$
K \varphi \leftrightarrow \neg B^{\neg \varphi} \top \leftrightarrow \neg B^{\neg \varphi} \neg \varphi
$$

which gives a rather unusual definition of knowledge in terms of conditional beliefs: this identity corresponds neither to the definition of knowledge in $[7,8]$ in terms of conditional beliefs nor to the definition of "necessity" in [52] in terms of doxastic 
conditionals (see also, e.g., [21]). Moreover, the first equivalence in (9) shows that the conditional belief operator is not a normal modality: it does not obey the Necessitation Rule, and in particular the formula $B^{\varphi} \top$ is not in general a validity. The second equivalence in (9) shows that in our theory the AGM Success Postulate $B^{\varphi} \varphi$ written in terms of conditional beliefs is not always valid. ${ }^{17}$ Ideally, we would like to have all the AGM postulates in the appropriate form stated in terms of conditional beliefs to be valid with respect to our semantics. However, one can show that while the AGM Postulates 2-6, written in terms of conditional beliefs, are valid with respect to the above semantics, the postulates 1,7 and 8 are not. ${ }^{18}$ The basic semantics for conditional beliefs is thus not optimal in capturing all of the AGM postulates for static belief revision. This motivates the search for an alternative semantics for conditional beliefs which captures more of the AGM postulates and is compatible with the notion of belief in Stalnaker's system.

Fortunately, as mentioned, the definition of extremally disconnected spaces presents the alternative semantics for conditional beliefs: the relativization of $\operatorname{Int}(\mathrm{Cl}(\mathrm{Int}))$ given in (8). We consider this semantics an improvement of the basic semantics, since, as we will see in Theorem 9, it is more successful in capturing the rationality postulates of AGM theory. This is why we refer to this semantics as the refined topological semantics for conditional beliefs and knowledge. Another, and simpler, possible justification for the so-called refined semantics of conditional belief is that it validates an equivalence that generalizes the one for belief in a natural way:

Proposition 8 The following equivalence is valid in all topological spaces with respect to the refined topological semantics for conditional beliefs and knowledge

$$
B^{\varphi} \psi \leftrightarrow K(\varphi \rightarrow\langle K\rangle(\varphi \wedge K(\varphi \rightarrow \psi)))
$$

Proof Follows immediately from the semantic clauses of conditional beliefs and knowledge.

This shows that, just like simple beliefs, conditional beliefs can be defined in terms of knowledge and this identity corresponds to the definition of the "conditional connective $\Rightarrow$ " in [21]. Moreover, as a corollary of Proposition 8, we obtain that the equivalences

$$
B^{\top} \psi \stackrel{(1)}{\leftrightarrow} K(\top \rightarrow\langle K\rangle(\top \wedge K(\top \rightarrow \psi)) \stackrel{(2)}{\leftrightarrow} K\langle K\rangle K \psi \stackrel{(3)}{\leftrightarrow}\langle K\rangle K \psi
$$

are valid in the class extremally disconnected spaces. ${ }^{19}$ Interestingly, unlike the case of simple belief, knowledge can be defined in terms of conditional belief:

\footnotetext{
${ }^{17}$ We refer to $[7,8]$ for the treatment of AGM theory in terms of conditional beliefs as a theory of static belief revision. Some of the axioms studied in [7,8] are stated in Theorem 9 and Proposition 10 with their names corresponding to the AGM postulates.

${ }^{18}$ The interested reader can find a more detailed discussion about this semantics in [46].

${ }^{19}$ In fact, equivalences (1) and (2) are valid in the class of all topological spaces, however, equivalence (3) is valid only in the class of extremally disconnected spaces.
} 
Proposition 9 The following equivalences are valid in all topological spaces with respect to the refined topological semantics for conditional beliefs and knowledge

$$
K \varphi \leftrightarrow B \neg \varphi \perp \leftrightarrow B^{\neg \varphi} \varphi .
$$

Proof Follows immediately from the semantic clauses of conditional beliefs and knowledge.

Proposition 9 constitutes another argument in favor of the refined semantics for conditional beliefs over the basic one: as also stated in [8], this identity coincides with the definition of "necessity" in [52] in terms of doxastic conditionals (see also, e.g., $[7,21])$.

Therefore, the language $\mathcal{L}_{K C B}$ of knowledge and conditional beliefs, $\mathcal{L}_{K B}$, and even the unimodal fragment of $\mathcal{L}_{K}$ having $K$ as the only modality and the unimodal fragment $\mathcal{L}_{C B}$ having only conditional belief modalities, have the same expressive power, since we can define simple beliefs and conditional beliefs in terms of knowledge (Proposition 5 and Proposition 8, respectively), and we can define simple beliefs and knowledge in terms of conditional beliefs (Proposition 8 and Proposition 9, respectively). As neither knowledge nor conditional beliefs can be defined in terms of simple beliefs, the unimodal fragment $\mathcal{L}_{B}$ having $B$ as the only modality is less expressive than the aforementioned systems (Fig. 3).

As for completeness, this can be obtained trivially:

Theorem 8 The logic $\mathbf{K C B}$ of knowledge and conditional beliefs is axiomatized completely by the system $\mathbf{S 4 . 2}$ for the knowledge modality $K$ together with the following equivalences:

1. $B^{\varphi} \psi \leftrightarrow K(\varphi \rightarrow\langle K\rangle(\varphi \wedge K(\varphi \rightarrow \psi)))$

2. $B \varphi \leftrightarrow B^{\top} \varphi$

The validity of (1) and (2) is given by Proposition 8 . While (2) reduces belief to conditional belief, (1) reduces conditional beliefs to knowledge. Hence, the proof of Theorem 8 follows from the topological completeness of S4.2.

Finally, we evaluate the success of the refined semantics in capturing the rationality postulates of AGM theory.

Fig. 3 Expressivity diagram with respect to topo-models

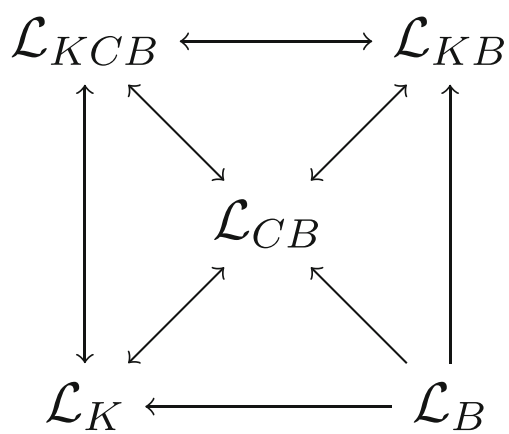


Theorem 9 The following formulas are valid in all topological spaces with respect to the refined topological semantics for conditional beliefs and knowledge

$\begin{array}{ll}\text { Normality: } & B^{\theta}(\varphi \rightarrow \psi) \rightarrow\left(B^{\theta} \varphi \rightarrow B^{\theta} \psi\right) \\ \text { Truthfulness of Knowledge: } & K \varphi \rightarrow \varphi \\ \text { Persistence of Knowledge: } & K \varphi \rightarrow B^{\theta} \varphi \\ \text { Strong Positive Introspection: } & B^{\theta} \varphi \rightarrow K B^{\theta} \varphi \\ \text { Success of Belief Revision: } & B^{\varphi} \varphi \\ \text { Consistency of Revision: } & \neg K \neg \varphi \rightarrow \neg B^{\varphi} \perp \\ \text { Inclusion: } & B^{\varphi \wedge \psi} \theta \rightarrow B^{\varphi}(\psi \rightarrow \theta) \\ \text { Cautious Monotonicity: } & B^{\varphi} \psi \wedge B^{\varphi} \theta \rightarrow B^{\varphi \wedge \psi} \theta\end{array}$

Moreover, the Necessitation rule for conditional beliefs:

$$
\text { From } \vdash \varphi \text { infer } \vdash B^{\psi} \varphi
$$

preserves validity.

Proof See Appendix B1.

The validity of the Normality principle and the Necessitation rule shows that, unlike in case of the basic topological semantics for conditional beliefs, the conditional belief modality is a normal modal operator with respect to the refined semantics. Moreover, the refined semantics also validates the Success Postulate. However, in this case, we have to restrict the principle of Consistency of Belief Revision to the formulas that are consistent with the agent's knowledge. This is in fact a desirable restriction taking into account the agent's knowledge and is perfectly compatible with the corresponding dynamic system that we will present in the next section. Intuitively, if the agent knows $\neg \varphi$ with some degree of certainty, she should not revise her beliefs with $\varphi$. As conditional beliefs pre-encode possible future belief changes of an agent and the future belief changes must be based on the new information consistent with the agent's knowledge, her consistent conditional beliefs must pre-encode the possibilities that are in fact consistent with her knowledge.

The failure of Strong Negative Introspection is an expected result for the following reasons. First of all, observe that Theorem 8 and Theorem 9 imply that all the formulas stated in Theorem 9 are theorems of the system KCB. Recall that

$$
\neg B^{\theta} \varphi \rightarrow K \neg B^{\theta} \varphi
$$

is the principle of Strong Negative Introspection. If this principle were a theorem of $\mathbf{K C B}$, then in particular $\neg B^{\neg \varphi} \varphi \rightarrow K \neg B \neg \varphi \varphi$ would be a theorem of $\mathbf{K C B}$. Then, by Proposition 9, we would obtain

$$
\neg K \varphi \rightarrow K \neg K \varphi
$$

as a theorem of KCB. However, Theorem 8 states that the knowledge modality of KCB is an S4.2-type modality implying that $\neg K \varphi \rightarrow K \neg K \varphi$ is not a theorem of the system.

Moreover, even the extremally disconnected spaces fail to validate Rational Monotonicity, which captures the AGM postulate of Superexpansion, with respect to the refined topological semantics for conditional beliefs and knowledge. However, a 
weaker principle, namely, the principle of Cautious Monotonicity is valid in all topological spaces. This principle says that if the agent would come to believe $\psi$ and would also come to believe $\theta$ if she would learn $\varphi$, her learning $\psi$ should not defeat her belief in $\theta$ and vice versa. In [36], the authors state that D. Gabbay also gives a convincing argument to accept Cautious Monotonicity: "if $\varphi$ is an enough reason to believe $\psi$ and also to believe $\theta$, then $\varphi$ and $\psi$ should also be enough to make us believe $\theta$, since $\varphi$ was enough anyway and, on this basis, $\psi$ was accepted" [36, p. 178].

The refined conditional belief semantics therefore captures the AGM postulates 1-7 together with a weaker version of 8 on all topological spaces. It is thus more successful than the basic one in modeling static belief change of a rational agent. Moreover, we will show in Section 4.2 that the refined semantics for conditional beliefs and knowledge validates Rational Monotonicity in a restricted class of topological spaces, namely in the class of hereditarily extremally disconnected spaces, and therefore it is able to capture the AGM postulates 1-8 stated in terms of conditional beliefs.

Summing up the work that has been done so far in this paper, a new topological semantics for belief on extremally disconnected spaces is proposed in [4, 46] and it has been proven, in this setting, that the complete logic of knowledge and belief is Stalnaker's system KB, the complete logic of knowledge is $\mathbf{S 4 . 2}$ and the complete logic of belief is KD45 in this setting. Moreover, we provided a semantics for conditional beliefs again on extremally disconnected spaces as well as complete axiomatizations of the corresponding static systems. These results on extremally disconnected spaces, however, encounter problems when extended to a dynamic setting by adding update modalities formalized as model restriction by means of subspaces.

\subsection{Dynamic Conditioning: Updates}

In DEL, update (dynamic conditioning) corresponds to change of beliefs through learning hard information. Unlike the case for conditional beliefs, update induces a global change in the model.

The most standard topological analogue of this corresponds to taking the restriction of a topology $\tau$ on $X$ to a subset $P \subseteq X$. This way, we obtain a subspace of a given topological space.

Topological Semantics for Update Modalities We now consider the language $\mathcal{L}_{! K C B}$ obtained by adding to the language $\mathcal{L}_{K C B}$ (existential) dynamic update modalities $\langle! \varphi\rangle \psi$ associated with updates. $\langle! \varphi\rangle \psi$ means that $\varphi$ is true and after the agent learns the new information $\varphi, \psi$ becomes true. The dual $[! \varphi]$ is defined as $\neg\langle! \varphi\rangle \neg$ as usual and $[! \varphi] \varphi$ means that if $\varphi$ is true then after the agent learns the new information $\varphi$, $\psi$ becomes true.

Given a topo-model $(X, \tau, v)$ and $\varphi \in \mathcal{L}_{! K C B}$, we denote by $\mathcal{M}_{\varphi}$ the restricted model $\mathcal{M}_{\varphi}=\left(\llbracket \varphi \rrbracket, \tau_{\llbracket \varphi \rrbracket}, v_{\llbracket \varphi \rrbracket}\right)$ where $\llbracket \varphi \rrbracket=\llbracket \varphi \rrbracket^{\mathcal{M}}, \tau_{\llbracket \varphi \rrbracket}=\{U \cap \llbracket \varphi \rrbracket \mid U \in \tau\}$ and $v_{\llbracket \varphi \rrbracket}(p)=v(p) \cap \llbracket \varphi \rrbracket$ for any $p \in$ Prop. Then, the semantics for the dynamic language $\mathcal{L}_{! K C B}$ is obtained by extending the semantics for $\mathcal{L}_{K C B}$ with:

$$
\llbracket\langle! \varphi\rangle \psi \rrbracket^{\mathcal{M}}=\llbracket \psi \rrbracket^{\mathcal{M}_{\varphi}} .
$$


Updates in general are expected to cause changes in an agent's knowledge and belief in some propositions, however, the way she reasons about her epistemic/doxastic state, in a sense the defining properties of the type of agent we consider, should remain unaffected. This amounts to saying that any restricted model should as well make the underlying static knowledge and belief logics sound. In particular, as we work with rational, highly idealized normal agents that hold consistent beliefs, we demand them not to lose these properties after an update with true information. With respect to the closure-interior semantics, these requirements are satisfied if and only if the resulting structure is extremally disconnected: under the topological belief semantics, both the axiom of Normality

$$
B(\varphi \wedge \psi) \leftrightarrow(B \varphi \wedge B \psi)
$$

and the axiom of Consistency of Belief

$$
B \varphi \rightarrow \neg B \neg \varphi
$$

characterize extremally disconnected spaces (see, Lemmas 4 and 5, respectively, in Appendix A.4). Therefore, if the restricted model is not extremally disconnected, the agent comes to have inconsistent beliefs after an update with hard true information. In order to avoid possible confusions, we note that $B \perp$ is never true with respect to the closure-interior semantics since $\llbracket B \perp \rrbracket=\mathrm{Cl}(\operatorname{Int}(\emptyset))=\emptyset$. By an agent having inconsistent beliefs, we mean that she believes mutually contradictory propositions such as $\varphi$ and $\neg \varphi$ at the same time, without in fact believing $B \perp$, as also illustrated by the following example:

Example Consider the Alexandroff topo-model $\mathcal{M}=(X, \tau, v)$ where $X=$ $\left\{x_{1}, x_{2}, x_{3}, x_{4}\right\}, \tau=\left\{X, \emptyset,\left\{x_{4}\right\},\left\{x_{2}, x_{4}\right\},\left\{x_{3}, x_{4}\right\},\left\{x_{2}, x_{3}, x_{4}\right\}\right\}$ and $V(p)=$ $\left\{x_{1}, x_{2}, x_{3}\right\}$ and $V(q)=\left\{x_{2}, x_{4}\right\}$ for some $p, q \in$ Prop. It is easy to see that $\mathcal{X}$ corresponds to a directed reflexive transitive relational frame as depicted in Fig. 4a, where the reflexive and transitive arrows are omitted. It is easy to check that $(X, \tau)$ is an extremally disconnected space and $B q \rightarrow \neg B \neg q$ is valid in $\mathcal{X}$. We stipulate that $x_{1}$ is the actual state and $p$ is truthfully announced. The updated (i.e., restricted) model is then $\mathcal{X}_{p}=\left(\llbracket p \rrbracket, \tau_{p}, V_{p}\right)$ where $\llbracket p \rrbracket=\left\{x_{1}, x_{2}, x_{3}\right\}$, $\tau_{p}=\left\{\llbracket p \rrbracket, \emptyset,\left\{x_{2}\right\},\left\{x_{3}\right\},\left\{x_{2}, x_{3}\right\}\right\}, V^{p}(p)=\left\{x_{1}, x_{2}, x_{3}\right\}$ and $V_{p}(q)=\left\{x_{2}\right\}$. Here, $\left(\llbracket p \rrbracket, \tau_{p}\right)$ is not an extremally disconnected space (similarly, the underlying Kripke frame is not directed) since $\left\{x_{3}\right\}$ is an open subset of $\left(\llbracket p \rrbracket, \tau_{p}\right)$ but $\mathrm{Cl}_{p}\left(\left\{x_{3}\right\}\right)=$ $\left\{x_{1}, x_{3}\right\}$ is not open in $\left(\llbracket p \rrbracket, \tau_{p}\right)$. Moreover, as $x_{1} \in \llbracket B q \rrbracket^{\mathcal{X}_{p}}=\mathrm{Cl}_{p}\left(\operatorname{Int}_{p}\left(\left\{x_{2}\right\}\right)\right)=$ $\left\{x_{1}, x_{2}\right\}$ and $x_{1} \in \llbracket B \neg q \rrbracket^{\mathcal{X}_{p}}=\mathrm{Cl}_{p}\left(\operatorname{Int}_{p}\left(\left\{x_{1}, x_{3}\right\}\right)\right)=\left\{x_{1}, x_{3}\right\}$, the agent comes to believe both $q$ and $\neg q$, implying that the restricted model falsifies (CB) at $x_{1}$. Consequently, it also falsifies $(\mathrm{K})$ since $\llbracket B(q \wedge \neg q) \rrbracket^{\mathcal{X}_{p}}=\emptyset$.

One possible solution for this problem is giving up on the (.2)-axiom for knowledge and extending the class of spaces we work with: we can focus on all topological spaces instead of working with only extremally disconnected spaces and provide semantics for belief in such a way that the aforementioned axioms which were problematic on extremally disconnected spaces would be valid on all topological spaces. This way, we do not need to worry about any additional topological property that is 


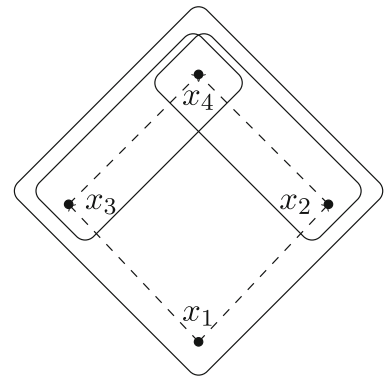

(a) $(X, \tau, \nu)$

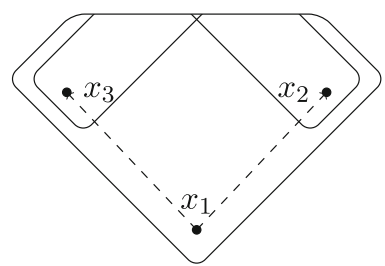

(b) $\left(\llbracket p \rrbracket, \tau^{p}, V^{p}\right)$

Fig. 4 Update of $(X, \tau, \nu)$ by $p$

supposed to be inherited by subspaces. This solution, however unsurprisingly, leads to a weakening of the underlying static logic of knowledge and belief. It is well known that the knowledge logic of all topological spaces under the interior semantics is $\mathbf{S} \mathbf{4}$ and we explored the (weak) belief logic of all topological spaces under the topological belief semantics in [5]. A more natural solution, however, would be to force the underlying static systems of knowledge and belief to be preserved after an update. One way to do this, we propose in this work, is to further restrict our attention to hereditarily extremally disconnected spaces, thereby, we guarantee that no model restriction leads to changes in the underlying static logics of knowledge and belief, and in particular, leads to inconsistent beliefs. As the logic of hereditarily extremally disconnected spaces under the interior semantics is $\mathbf{S 4 . 3}$, the underlying static logic, in this case, would consist in $\mathbf{S 4 . 3}$ as the logic of knowledge but again KD45 as the logic of belief (see Theorems 3 and 10 below).

This solution to the above mentioned problem of the dynamic setting based on extremally disconnected spaces (or, equivalently stated, based on an S4.2-type static knowledge) is also motivated by the theory of subframe logics. The fact that extremally disconnected spaces are not closed under subspaces corresponds to $\mathbf{S 4 . 2}$ not being a subframe logic (see [22, Section 9.4]). Similarly, the logical counterpart of the fact that hereditarily extremally disconnected spaces are extremally disconnected spaces closed under subspaces is that the subframe closure of $\mathbf{S 4 . 2}$ is $\mathbf{S 4 . 3}$, see [70, Section 4.7]. This leads to two important conclusions. Firstly, given that updates we consider are interpreted by means of subspaces, the transition from the static base $\mathbf{S 4 . 2}$ to $\mathbf{S 4 . 3}$ is the least strengthening we could use in order to obtain a logic of knowledge that behaves well when extended with updates. Secondly, working with hereditarily extremally disconnected spaces amounts to working with S4.2type of agent that keeps being $\mathbf{S 4 . 2}$ whenever she receives new information. Recall that updates are expected to cause changes in an agent's knowledge and belief in some propositions, however, the way she reasons about her epistemic/doxastic state, in a sense the defining properties of the type of agent we consider, should remain unaffected. Therefore, our rather technically looking solution to the aforementioned problem in fact amounts to having an agent of S4.2-type who keeps holding S4.2-type 
knowledge when faced with new truthful information. And, as explained above, S4.2type knowledge that is resistant to updates is in fact of S4.3-type. Following [38], we could then argue that some previous work, e.g. [40, 53, 67], settled for $\mathbf{S 4 . 2}$ since they did not consider the behavior of $\mathbf{S 4 . 2}$ in a dynamic setting: the logic of knowledge of an S4.2-type agent who learns new information via updates is essentially $\mathbf{S 4 . 3}$.

As Stalnaker also observed in [53], the derived logic of belief with belief modality defined as epistemic possibility of knowledge, i.e., as $\langle K\rangle K$, is KD45 in case $K$ is an S4.3 modality:

Theorem 10 In the topological belief semantics, $\mathrm{KD} 45$ is the complete logic of belief with respect to the class of hereditarily extremally disconnected spaces.

Proof See Appendix A.5 and observe that topological spaces corresponding to pins are in fact hereditarily extremally disconnected.

Then, we again obtain a complete logic $\mathbf{K C B}^{\prime}$ of knowledge and conditional beliefs trivially, yet, with respect to the class of hereditarily extremally disconnected spaces:

Theorem 11 The logic $\mathbf{K C} \boldsymbol{B}^{\prime}$ of knowledge and conditional beliefs is axiomatized completely by the system $\mathbf{S 4 . 3}$ for the knowledge modality $K$ together with the following equivalences:

1. $B^{\varphi} \psi \leftrightarrow K(\varphi \rightarrow\langle K\rangle(\varphi \wedge K(\varphi \rightarrow \psi)))$

2. $B \varphi \leftrightarrow B^{\top} \varphi$

Proposition 10 The following formula

$$
B^{\varphi}(\psi \rightarrow \theta) \wedge \neg B^{\varphi} \neg \psi \rightarrow B^{\varphi \wedge \psi} \theta,
$$

called the axiom of Rational Monotonicity for conditional beliefs, is valid on hereditarily extremally disconnected spaces.

Proof See Appendix B.1 and use the fact that $\mathbf{S 4 . 3}$ is sound and complete with respect to reflexive, transitive and linear Kripke frames.

We can then conclude, by Theorem 9 and Proposition 10, that the refined semantics for conditional beliefs on hereditarily extremally disconnected spaces captures the AGM postulates 1-8.

We now implement updates on hereditarily extremally disconnected spaces and show that the problems occurred when we work with extremally disconnected spaces do not arise here: we in fact obtain a complete dynamic logic of knowledge and conditional beliefs with respect to the class of hereditarily extremally disconnected spaces. We again consider the language $\mathcal{L}_{! K C B}$ and semantics for update modalities $\langle! \varphi\rangle \psi$ by means of subspaces exactly the same way as formalized in the beginning of the current section, i.e., by using the restricted model $\mathcal{M}_{\varphi}$ with the semantic clause

$$
\llbracket\langle! \varphi\rangle \psi \rrbracket^{\mathcal{M}}=\llbracket \psi \rrbracket^{\mathcal{M}_{\varphi}} .
$$


In this setting, however, as the underlying static logic $\mathbf{K C} \mathbf{B}^{\prime}$ is the logic of hereditarily extremally disconnected spaces, we implement updates on those spaces. Since the resulting restricted model $\mathcal{M}_{\varphi}$ is always based on a hereditarily extremally disconnected (sub)space, we do not face the problem of loosing some validities of the corresponding static system: all the axioms of $\mathbf{K C B}$ (and, in particular, of $\mathbf{S 4 . 3}$ and KD45) will still be valid in the restricted space.

Against this static background, we can further axiomatize the logic of public announcements, knowledge and conditional beliefs, following the standard DELtechnique: This is done by adding to $\mathbf{K C B}^{\prime}$ a set of reduction axioms that give us a recursive rewriting algorithm to step-by-step translate every formula containing public announcement modalities to a provably equivalent formula in the static language. The completeness of the dynamic system then follows from the soundness of the reduction axioms and the completeness of the underlying static logic (see, e.g., [64, Section 7.4] for a detailed presentation of completeness by reduction, and see [68] for an elaborate discussion of axiomatizations of public announcement logics).

Theorem 12 The complete and sound dynamic logic !KCB' of knowledge and conditional beliefs with respect to the class of hereditarily extremally disconnected spaces is obtained by adding the following reduction axioms to any complete axiomatization of the logic $\boldsymbol{K} \boldsymbol{C B}$ :
1. $\langle! \varphi\rangle p \leftrightarrow(\varphi \wedge p)$
2. $\langle! \varphi\rangle \neg \psi \leftrightarrow(\varphi \wedge \neg\langle! \varphi\rangle \psi)$
3. $\langle! \varphi\rangle(\psi \wedge \theta) \leftrightarrow(\langle! \varphi\rangle \psi \wedge\langle! \varphi\rangle \theta)$
4. $\langle! \varphi\rangle K \psi \leftrightarrow(\varphi \wedge K(\varphi \rightarrow\langle! \varphi\rangle \psi))$
5. $\langle! \varphi\rangle B^{\theta} \psi \leftrightarrow\left(\varphi \wedge B^{\langle! \varphi\rangle \theta}\langle! \varphi\rangle \psi\right)$
6. $\langle! \varphi\rangle\langle! \psi\rangle \chi \leftrightarrow\langle!\langle! \varphi\rangle \psi\rangle \chi$

\section{Conclusion and Future Work}

Summary In this work, we proposed a new topological semantics for belief in terms of the closure of the interior operator. Our proposal provides an intuitive interpretation of Stalnaker's conception of (full) belief as subjective certainty due to the nature of topological spaces, in particular, through the definitions of interior and closure operators as elaborated in Section 3.3.1.

The majority of approaches to knowledge and belief take belief-the weaker notion-as basic and then strengthen it to obtain a "good" concept of knowledge. Our work provides a semantics for Stalnaker's system which approaches the issue from the other direction, i.e., taking knowledge as primitive. The formal setting developed in our studies therefore adds a precise semantic framework to a rather non-standard approach to knowledge and belief, providing a new semantics to Stalnaker's system and imparting if not additional momentum at least an additional interpretation of it.

Furthermore, we explore topological analogues of static and dynamic conditioning by providing a topological semantics for conditional belief and update modalities. 
We evaluated two, basic and refined, topological semantics for conditional beliefs directly obtained from the semantics of simple belief by conditioning and argued that the latter is an improvement of the former. We demonstrated that the refined semantics for conditional beliefs quite successfully captures the rationality postulates of AGM theory: it validates the appropriate versions of the AGM postulates 1-7 and a weaker version of postulate 8 (see Theorem 9). We moreover gave a complete axiomatization of the logic of conditional beliefs and knowledge. Although the semantics proposed for the aforementioned static notions (namely; knowledge, (full) belief and conditional beliefs) completely captures their intended meanings, modelling these notions on extremally disconnected spaces causes the problem of preserving the important structural properties of these spaces given that extremally disconnectedness is not a hereditary property as explained in Section 4.2 and also stated in [5]. In this paper, we solved this problem by restricting the class of spaces we work with to the class of hereditarily extremally disconnected spaces and we formalized knowledge, belief and conditional beliefs also on hereditarily extremally disconnected spaces together with updates and provide complete axiomatizations for the corresponding logics. As a result of working on hereditarily extremally disconnected spaces, the unimodal logic of knowledge is strengthened from S4.2 (the epistemic logic of extremally disconnected spaces) to $\mathbf{S 4 . 3}$ (the epistemic logic of hereditarily extremally disconnected spaces), whereas the unimodal logic of belief remains to be KD45. We also showed that hereditarily extremally disconnected spaces validate the AGM axiom 8, stated as Rational Monotonicity in terms of conditional beliefs, and concluded that our topological semantics can capture the theory of belief revision $\mathrm{AGM}$ as a static one formalized in a modal setting in terms of conditional beliefs.

Relevant and Future Research At a high level, this paper takes a further small step toward developing a satisfactory epistemic/doxastic formal framework in which we can talk about evidential grounds of knowledge and belief. It does so by extending the interior-based topological semantics for knowledge by a semantic clause for belief, which arguably works better than the previous proposal based on the coderived set operator. However, within the current setting, everything we can say about evidence has to be said at a purely semantic level (see Sections 2.3 and 3.3.1 to recall the topological, evidence-related readings of knowledge and belief, respectively). As we have not yet introduced any "evidence modalities", the modal language cannot really say anything concerning the link between evidence and belief, or evidence and knowledge. We explored topological semantics for evidence and its connection to topological (evidence-based) knowledge and belief in [6] by building topological evidence models generalizing those of van Benthem and Pacuit [62] and also interpret evidence dynamics on such models following the aforementioned work.

In this paper, we focused on providing a topological semantics for single agent logics for knowledge, belief, conditional beliefs and updates. However, reasoning 
about knowledge, belief and especially about information change becomes particularly interesting when applied to multi-agent cases. One natural continuation of this work therefore consists in extending our framework to a multi-agent setting and providing topological semantics for operators, such as common knowledge and common belief, in line with, e.g., [54, 63].

Acknowledgements Nick Bezhanishvili was partially supported by Shota Rustaveli National Science Foundation grant \# DI-2016-25. Aybüke Özgün's research was funded by the European Research Council (ERC CoG), Consolidator grant no. 681404, 'The Logic of Conceivability'. Sonja Smets' contribution to this paper has received funding from the European Research Council under the European Community's 7th Framework Programme/ERC Grant agreement no. 283963. This work was presented (in part) at the following workshops and conferences: The Fourth International Workshop on Logic, Rationality and Interaction (LORI 2013); The Second LogiCIC Workshop-Social Dynamics of Information Chance (LogiCIC 2013); Epistemic Logic for Individual, Social, and Interactive Epistemology Workshop (ELISIEM 2014) and International Workshop on Topological Methods in Logic IV (ToLo 2014). We are grateful to the conference organizers and participants who have provided us with valuable feedback on this work in the last years, and two anonymous referees of this journal for helpful comments and remarks.

Open Access This article is distributed under the terms of the Creative Commons Attribution 4.0 International License (http://creativecommons.org/licenses/by/4.0/), which permits unrestricted use, distribution, and reproduction in any medium, provided you give appropriate credit to the original author(s) and the source, provide a link to the Creative Commons license, and indicate if changes were made.

\section{Appendix A: The Topology of Full Belief and Knowledge}

\section{A.1 Proof of Proposition 6}

Observe that for any topo-model $(X, \tau, v)$ and for any $\varphi, \psi \in \mathcal{L}_{K B}$,

$$
\llbracket \varphi \rightarrow \psi \rrbracket=X \text { iff } \llbracket \varphi \rrbracket \subseteq \llbracket \psi \rrbracket .
$$

Let $(X, \tau)$ be a topological space and $v$ be an arbitrary valuation on $(X, \tau)$. We know, by the soundness of $\mathbf{S 4}$ under the interior semantics, that the axioms $(\mathrm{K}),(\mathrm{T}),(\mathrm{KK})$ and the inference rules of $\mathbf{K B}$ are valid in all topological spaces. In addition, (NI), $(\mathrm{KB})$ and $(\mathrm{FB})$ are also valid in all topological spaces. Here, we demonstrate only the proof for the validity of (NI):

$$
\begin{aligned}
X=\llbracket \neg B \varphi \rightarrow K \neg B \varphi \rrbracket & \text { iff } \llbracket \neg B \varphi \rrbracket \subseteq \llbracket K \neg B \varphi \rrbracket \\
& \text { iff } X \backslash(\mathrm{Cl}(\operatorname{Int}(\llbracket \varphi \rrbracket))) \subseteq \operatorname{Int}(X \backslash(\mathrm{Cl}(\operatorname{Int}(\llbracket \varphi \rrbracket)))) \\
& \text { iff } \operatorname{Int}(\mathrm{Cl}(X \backslash \llbracket \varphi \rrbracket)) \subseteq \operatorname{Int}(\operatorname{Int}(\mathrm{Cl}(X \backslash \llbracket \varphi \rrbracket)))
\end{aligned}
$$

Since $\operatorname{Int}(\mathrm{Cl}(X \backslash \llbracket \varphi \rrbracket))=\operatorname{Int}(\operatorname{Int}(\mathrm{Cl}(X \backslash \llbracket \varphi \rrbracket)))$ is true in all topological spaces (by (I4) in Table 1), the result follows. The proofs for the validity of the axioms (KB) and (FB) follow similarly. 
Moreover, both (CB) and (PI) are valid on $(X, \tau)$ (under our proposed semantics) iff $(X, \tau)$ is extremally disconnected. Here, we write only the proof for the validity of $(\mathrm{CB})$, the validity of (PI) follows similarly:

$$
\begin{aligned}
X=\llbracket B \varphi \rightarrow \neg B \neg \varphi \rrbracket & \text { iff } \llbracket B \varphi \rrbracket \subseteq \llbracket\langle B\rangle \varphi \rrbracket \\
& \text { iff } \mathrm{Cl}(\operatorname{Int}(\llbracket \varphi \rrbracket)) \subseteq \operatorname{Int}(\mathrm{Cl}(\llbracket \varphi \rrbracket)) \\
& \text { iff } \mathrm{Cl}(\operatorname{Int}(\llbracket \varphi \rrbracket))=\operatorname{Int}(\mathrm{Cl}(\operatorname{Int}(\llbracket \varphi \rrbracket))) \\
& \text { iff }(X, \tau) \text { is extremally disconnected. }
\end{aligned}
$$

Therefore, $(X, \tau)$ validates the axioms and rules of $\mathbf{K B}$ iff it is extremally disconnected.

\section{A.2. Proof of Theorem 4}

$(\Leftarrow) \quad$ This direction is proven in Proposition 6 .

$(\Rightarrow)$ Suppose $(X, \mathrm{~K}, \mathrm{~B})$ validates all the axioms and rules of $\mathbf{K B}$. Then, the validity of the $\mathbf{S 4}$ axioms implies that $\mathrm{K}$ satisfies the Kuratowski conditions for topological interior, and so it gives rise to a topology $\tau$ in which $\mathrm{K}=\mathrm{Int}$, by the Theorem 5.3 in [25, p. 74] (see also Proposition 1.2.9 in [26, p. 23]). Then, since $(X, \mathrm{~K}, \mathrm{~B})$ validates all the axioms of $\mathbf{K B}$, we have $\llbracket B \varphi \leftrightarrow$ $\langle K\rangle K \varphi \rrbracket^{\mathcal{M}}=X$ for any model $\mathcal{M}=(X, \mathrm{~K}, \mathrm{~B}, v)$ and for all $\varphi \in \mathcal{L}_{K B}$ (by Proposition 5). Hence, $\llbracket B \varphi \rrbracket^{\mathcal{M}}=\mathrm{B} \llbracket \varphi \rrbracket^{\mathcal{M}}=\mathrm{Cl}\left(\operatorname{Int}\left(\llbracket \varphi \rrbracket^{\mathcal{M}}\right)\right.$, i.e., $\mathrm{B}=\mathrm{Cl}(\mathrm{Int})$. Thus, $(X, \mathrm{~K}, \mathrm{~B})=\left(X, \mathrm{~K}_{\tau}, \mathrm{B}_{\tau}\right)$ where $\tau$ is the topology generated by Int, $\mathrm{K}_{\tau}=\mathrm{Int}$ and $\mathrm{B}_{\tau}=\mathrm{Cl}(\mathrm{Int})$. Finally, the validity of the axiom (CB) proves that $(X, \tau)$ is extremally disconnected (see the proof of Proposition 6).

\section{A.3 Proof of Theorem 5}

Since axioms of $\mathbf{K B}$ are Sahlqvist formulas, $\mathbf{K B}$ is canonical, hence, complete with respect to its canonical model. However, the canonical model of $\mathbf{K B}$ is in fact an extensional model validating all of its axioms. Thus, by Topological Representation Theorem (Theorem 4 in Section 3.2), we have that KB is sound and complete with respect to the class of extremally disconnected spaces.

\section{A.4 Proof of Theorem 6}

Lemma 1 For any topo-model $\mathcal{M}=(X, \tau, v)$ and any $\varphi \in \mathcal{L}_{B}$ we have

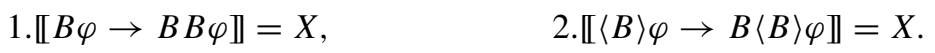

Proof Follows from basic properties of the interior and closure operators.

It follows from Lemma 1 that all topological spaces validate the axioms (4) and (5) under the topological belief semantics. However, the K-axiom $B \varphi \wedge B \psi \leftrightarrow B(\varphi \wedge \psi)$ 
and the D-axiom $B \varphi \rightarrow\langle B\rangle \varphi$ are not valid on all topological spaces but are valid on all extremally disconnected spaces:

Lemma 2 For any topological space $(X, \tau)$, we have $U \cap \mathrm{Cl}(A) \subseteq \mathrm{Cl}(U \cap A)$ for all $U \in \tau$ and $A \subseteq X$.

Proof Let $(X, \tau)$ be a topological space, $U \in \tau, A \subseteq X$ and $x \in X$. Suppose $x \in U \cap \mathrm{Cl}(A)$. Since $x \in \mathrm{Cl}(A)$, for all open neighbourhoods $V$ of $x, V \cap A \neq \emptyset$. Let $W$ be an open neightbourhood of $x$. Then, since $\tau$ is closed under finite intersection and $x$ is an element of both $W$ and $U$, the set $W \cap U$ is an open neighbouhood of $x$ as well. Thus, by the assumption that $x \in \mathrm{Cl}(A),(W \cap U) \cap A \neq \emptyset$, i.e., $W \cap(U \cap A) \neq \emptyset$. As $W$ has been chosen arbitrarily, $x \in \mathrm{Cl}(U \cap A)$.

Lemma 3 [44] The following are equivalent for a topological space $(X, \tau)$ :

1. $(X, \tau)$ is extremally disconnected.

2. $\mathrm{Cl}(U) \cap \mathrm{Cl}(V)=\mathrm{Cl}(U \cap V)$ for all $U, V \in \tau$.

3. $\mathrm{Cl}(U) \cap \mathrm{Cl}(V)=\emptyset$ for all $U, V \in \tau$ with $U \cap V=\emptyset$.

Lemma 4 A topological space $(X, \tau)$ validates the $\mathrm{K}$-axiom iff $(X, \tau)$ is extremally disconnected.

Proof Let $(X, \tau)$ be a topological space and $\mathcal{M}=(X, \tau, \nu)$ be a topo-model on $(X, \tau)$. Then,

$$
\begin{aligned}
& \quad X=\llbracket B \varphi \wedge B \psi \leftrightarrow B(\varphi \wedge \psi) \rrbracket \\
& \text { iff } \llbracket B \varphi \wedge B \psi \rrbracket=\llbracket B(\varphi \wedge \psi) \rrbracket \\
& \text { iff } \mathrm{Cl}(\operatorname{Int}(\llbracket \varphi \rrbracket)) \cap \mathrm{Cl}(\operatorname{Int}(\llbracket \psi \rrbracket))=\mathrm{Cl}(\operatorname{Int}(\llbracket \varphi \rrbracket \cap \llbracket \psi \rrbracket)) \\
& \text { iff } \mathrm{Cl}(\operatorname{Int}(\llbracket \varphi \rrbracket)) \cap \mathrm{Cl}(\operatorname{Int}(\llbracket \psi \rrbracket))=\mathrm{Cl}(\operatorname{Int}(\llbracket \varphi \rrbracket) \cap \operatorname{Int}(\llbracket \psi \rrbracket)) \\
& \text { iff }(X, \tau) \text { is extremally disconnected } \quad \text { (by), Table 1) }
\end{aligned}
$$

Lemma 5 A topological space $(X, \tau)$ validates the $\mathrm{D}$-axiom iff $(X, \tau)$ is extremally disconnected.

Proof See Appendix A.1, the case for (CB).

It follows from Lemmas 4 and 5 that the K-axiom and the D-axiom are not only valid on extremally disconnected spaces, they also characterize extremally disconnected spaces (under the topological belief semantics). Hence, the class of extremally disconnected spaces is the largest class of topological spaces which validates the Kaxiom and the D-axiom. The fact that both $\mathrm{K}$-axiom and the D-axiom characterizing extremally disconnectedness might seem surprising at first sight. However, given that we interpret the knowledge modality $K$ as the interior and the belief modality $B$ as the closure of the interior operators on topological spaces, we obtain that the formula $(B \varphi \rightarrow\langle B\rangle \varphi)$ is equivalent to $(\langle K\rangle K \varphi \rightarrow K\langle K\rangle \varphi)$, which is the (.2)-axiom, and the formula $(B \varphi \wedge B \psi \leftrightarrow B(\varphi \wedge \psi))$ is equivalent to $(\langle K\rangle K \varphi \wedge\langle K\rangle K \psi \leftrightarrow$ 
$\langle K\rangle K(\varphi \wedge \psi))$. Thus, the above propositions only state that a topological space validates $(\langle K\rangle K \varphi \rightarrow K\langle K\rangle \varphi)$ iff it validates $(\langle K\rangle K \varphi \wedge\langle K\rangle K \psi \leftrightarrow\langle K\rangle K(\varphi \wedge \psi))$ iff it is extremely disconnected. In fact, Lemmas 1, 4 and 5 yield the soundness of KD45, i.e., they prove Theorem 6.

\section{A.5 Proof of Theorem 7}

For the topological completeness proof of KD45 we make use of the completeness of KD45 with respect to the standard Kripke semantics. We first recall some frame conditions concerning the relational completeness of the corresponding systems. Let $(X, R)$ be a transitive Kripke frame. A non-empty subset $\mathcal{C} \subseteq X$ is a cluster if

(1) for each $x, y \in \mathcal{C}$ we have $x R y$, and

(2) there is no $D \subseteq X$ such that $\mathcal{C} \subset D$ and $D$ satisfies (1).

A point $x \in X$ is called a maximal point if there is no $y \in X$ such that $x R y$ and $\neg(y R x)$. We call a cluster a final cluster if all its points are maximal. It is not hard to see that for any final cluster $\mathcal{C}$ of $(X, R)$ and any $x \in \mathcal{C}$, we have $R(x)=\mathcal{C}$. A transitive Kripke frame $(X, R)$ is called cofinal if it has a unique final cluster $\mathcal{C}$ such that for each $x \in X$ and $y \in \mathcal{C}$ we have $x R y$. We call a cofinal frame a brush if $X \backslash \mathcal{C}$ is an irreflexive antichain, i.e., for each $x, y \in X \backslash \mathcal{C}$ we have $\neg(x R y)$ where $\mathcal{C}$ is the final cluster. A brush with a singleton $X \backslash \mathcal{C}$ is called a pin. By definition, every brush and every pin is transitive. Finally, a transitive frame $(X, R)$ is called rooted, if there is an $x \in X$, called a root, such that for each $y \in X$ with $x \neq y$ we have $x R y$. Hence, every rooted brush is in fact a pin. Figure 5 illustrates brushes and pins, respectively.

For the proof of the following lemma see, e.g., [22, Chapter 5] and [19, Chapters $2,4]$.

Lemma 6 KD45 is a sound and complete with respect to the class of brushes, and with respect to the class of pins. In fact, $\mathbf{K D 4 5}$ is sound and complete with respect to the class of finite pins.

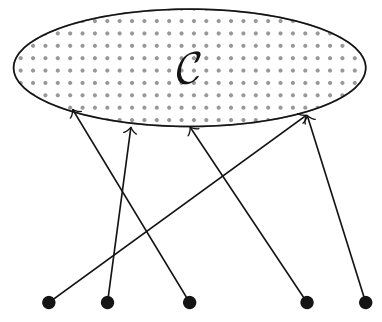

(a) Brush

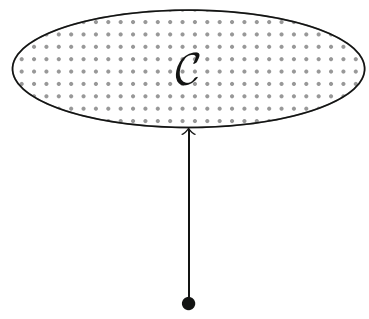

(b) Pin

Fig. 5 An example of a brush and of a pin, where the top ellipses illustrate the final clusters and an arrow relates the state it started from to every element in the cluster 
Similar to the construction in Section 2.2.2, we can build an Alexandroff extremally disconnected space from a given pin. The only extra step consists in taking the reflexive closure of the initial pin. More precisely, for any frame $(X, R)$, let $R^{+}$denote the reflexive closure of $R$, defined as

$$
R^{+}=R \cup\{(x, x) \mid x \in X\} .
$$

Given a pin $(X, R)$, the set $\tau_{R^{+}}=\left\{R^{+}(x) \mid x \in X\right\}$ constitutes a topology on $X$. In fact, in this special case of pins, we have $\tau_{R^{+}}=\{X, \mathcal{C}, \emptyset\}$ where $\mathcal{C}$ is the final unique cluster of $(X, R)$. Therefore, it is easy to see that $\left(X, \tau_{R^{+}}\right)$is an Alexandroff extremally disconnected space. This construction leads to a natural correspondence between pins and Alexandroff extremally disconnected spaces. Moreover, any two such models $M=(X, R, v)$ and $\mathcal{M}_{\tau_{R^{+}}}=\left(X, \tau_{R^{+}}, v\right)$ make the same formulas of $\mathcal{L}_{B}$ true at the same states, as shown in Proposition 7.

Lemma 7 For all $\varphi \in \mathcal{L}_{B}$, any Kripke model $M=(X, R, v)$ based on a pin

$$
\|\varphi\|^{M}=\llbracket \varphi \rrbracket^{\mathcal{M}_{\tau_{R^{+}}}} .
$$

Proof The proof follows by subformula induction on $\varphi$; cases for the propositional variables and the Boolean connectives are elementary. So assume inductively that the result holds for $\psi$; we must show that it holds also for $\varphi:=B \psi$.

$(\Rightarrow)$ Suppose $M, x \models B \psi$, i.e., $R(x) \subseteq\|\psi\|^{M}$. Since $R(x)=\mathcal{C}$, we obtain

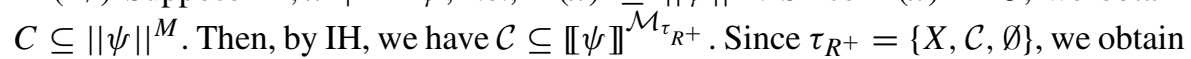
$\mathrm{Cl}(\operatorname{Int}(\mathcal{C}))=\mathrm{Cl}\left(\operatorname{Int}\left(\llbracket \psi \rrbracket^{\mathcal{M}_{\tau_{R^{+}}}}\right)\right)=X$. Therefore, $x \in \mathrm{Cl}\left(\operatorname{Int}\left(\llbracket \psi \rrbracket^{\mathcal{M} \tau_{R^{+}}}\right)\right)=X$, i.e.,

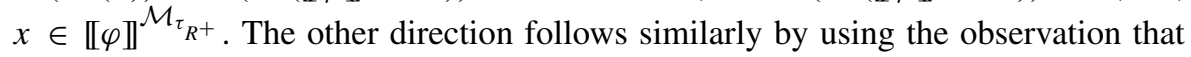
for all $A \subseteq X$ with $A \subset \mathcal{C}, \operatorname{Int}(A)=\emptyset$.

Theorem 7. In the topological belief semantics, KD45 is the complete logic of belief with respect to the class of extremally disconnected spaces.

Proof Let $\varphi \in \mathcal{L}_{B}$ such that $\varphi \notin \mathbf{K D 4 5}$. Then, by Lemma 6, there exists a relational model $M=(X, R, v)$, where $(X, R)$ is a pin, and $x \in X$ such that $M, x \notin \varphi$. Therefore, by Proposition 7, we obtain $\mathcal{M}_{\tau_{R^{+}}}, x \not \models \varphi$. Since $\mathcal{M}_{\tau_{R^{+}}}$is extremally disconnceted, we obtain the desired result.

\section{A.6 Proof of Proposition 7}

Let $(X, \tau)$ be a $D S O$-space and $U \in \tau$. Recall that for any $A \subseteq X, \operatorname{Cl}(A)=$ $d(A) \cup A$. So $\mathrm{Cl}(U)=d(U) \cup U$. Since $(X, \tau)$ is a $D S O$-space, $d(U)$ is an open subset of $X$. Thus, since $U$ is open as well, $\mathrm{Cl}(U)$ is open. Therefore, $(X, \tau)$ is an extremally disconnected space.

Now consider the topological space $(X, \tau)$ where $X=\{1,2,3\}$ and $\tau=$ $\{X, \emptyset,\{2\},\{1,2\}\}$. It is easy to check that for all $U \in \tau, \mathrm{Cl}(U)$ is open (in fact, for each $U \in \tau$ with $U \neq \emptyset, \mathrm{Cl}(U)=X)$. Hence, $(X, \tau)$ is an extremally disconnected space. However, as $\mathrm{Cl}(X \backslash\{2\})=\{1,3\}$, we have $2 \notin d(X)$. Thus, $(X, \tau)$ is not dense in itself and thus not a $D S O$-space. 


\section{Appendix B: Topological Models for Belief Revision: Static and Dynamic Conditioning}

\section{B.1 Proof of Theorem 9}

By Proposition 8, we know that each of the axioms can be rewritten by using only the knowledge modality $K$. We also know that the logic of knowledge $\mathbf{S 4}$ is complete with respect to to the class of reflexive and transitive Kripke frames. Therefore, we can show that each of the axioms is a theorem of $\mathbf{S 4}$ by using Kripke frames and the relational completeness of $\mathbf{S 4}$. Then, we can conclude that these axioms are also valid on all topological spaces, since $\mathbf{S} \mathbf{4}$ is sound with respect to the class of all topological spaces under the interior semantics. Recall that the semantic clauses for knowledge in the interior semantics and in the refined topological semantics for conditional beliefs and knowledge coincide. Here we only show the case for Cautious Monotonicity, the others follow similarly and can be found in [46].

Let $(X, R)$ be a reflexive and transitive Kripke frame, $M=(X, R, v)$ a model on $(X, R)$ and $x$ any element of $X$. By Proposition 8 , we can rewrite the axiom of Cautious Monotonicity as

$$
\begin{aligned}
K(\varphi & \rightarrow\langle K\rangle(\varphi \wedge K(\varphi \rightarrow \psi))) \wedge K(\varphi \rightarrow\langle K\rangle(\varphi \wedge K(\varphi \rightarrow \theta))) \\
& \rightarrow K((\varphi \wedge \psi) \rightarrow\langle K\rangle((\varphi \wedge \psi) \wedge K((\varphi \wedge \psi) \rightarrow \theta))) .
\end{aligned}
$$

Suppose $x \in\|K(\varphi \rightarrow\langle K\rangle(\varphi \wedge K(\varphi \rightarrow \psi))) \wedge K(\varphi \rightarrow\langle K\rangle(\varphi \wedge K(\varphi \rightarrow \theta)))\|$. Then,

$$
\begin{aligned}
& R(x) \subseteq\|\varphi \rightarrow\langle K\rangle(\varphi \wedge K(\varphi \rightarrow \psi))\|, \text { and } \\
& R(x) \subseteq\|\varphi \rightarrow\langle K\rangle(\varphi \wedge K(\varphi \rightarrow \theta))\|
\end{aligned}
$$

We want to show that $R(x) \subseteq\|(\varphi \wedge \psi) \rightarrow\langle K\rangle((\varphi \wedge \psi) \wedge K((\varphi \wedge \psi) \rightarrow \theta))\|$

Let $y \in R(x)$ such that $y \in\|\varphi \wedge \psi\|$. Then, by Eqs. 11 and 12, we have $y \in$ $\|\langle K\rangle(\varphi \wedge K(\varphi \rightarrow \psi))\|$ and $y \in\|\langle K\rangle(\varphi \wedge K(\varphi \rightarrow \theta))\|$, respectively. These imply there exists a $z_{0} \in X$ with $y R z_{0}$ such that

$$
z_{0} \in\|\varphi \wedge K(\varphi \rightarrow \psi)\|
$$

and there exists a $z_{1} \in X$ with $z_{1} R y$ such that

$$
z_{1} \in\|\varphi \wedge K(\varphi \rightarrow \theta)\|
$$

Hence, as $R$ is reflexive, we have $z_{0} \in\|\psi\|$ and thus $z_{0} \in\|\varphi \wedge \psi\|$ by Eq. 13. Then, since $x R y R z_{0}$ and $R$ is transitive, we have $z_{0} \in\|\varphi \rightarrow\langle K\rangle(\varphi \wedge K(\varphi \rightarrow \theta))\|$, by Eq. 12. Thus, as $z_{0} \in\|\varphi\|$, we obtain $z_{0} \in\|\langle K\rangle(\varphi \wedge K(\varphi \rightarrow \theta))\|$. This implies that these is a $z_{2} \in X$ with $z_{0} R z_{2}$ such that $z_{2} \in\|\varphi \wedge K(\varphi \rightarrow \theta)\|$. Then, since $R$ is transitive and $z_{0} \in\|K(\varphi \rightarrow \psi)\|$, we have $z_{2} \in\|K(\varphi \rightarrow \psi)\|$. Hence, since $R$ is reflexive and $z_{2} \in\|\varphi\|$, we get $z_{2} \in\|\psi\|$ implying that $z_{2} \in\|\varphi \wedge \psi\|$. Moreover, $z_{2} \in\|K(\varphi \rightarrow \psi)\|$ and $z_{2} \in\|K(\varphi \rightarrow \theta)\|$ imply that $z_{2} \in\|K((\varphi \wedge \psi) \rightarrow \theta)\|$. Therefore, $z_{2} \in\|(\varphi \wedge \psi) \wedge K((\varphi \wedge \psi) \rightarrow \theta)\|$. Hence, as $y R z_{0} R z_{2}$ and $R$ is transitive, $y \in\|\langle K\rangle((\varphi \wedge \psi) \wedge K((\varphi \wedge \psi) \rightarrow \theta))\|$. Hence, $y \in \|(\varphi \wedge \psi) \rightarrow$ 
$\langle K\rangle((\varphi \wedge \psi) \wedge K((\varphi \wedge \psi) \rightarrow \theta)) \|$. Sinca $y$ has been chosen arbitrarily from $R(x)$, we have $R(x) \subseteq\|(\varphi \wedge \psi) \rightarrow\langle K\rangle((\varphi \wedge \psi) \wedge K((\varphi \wedge \psi) \rightarrow \theta))\|$, i.e.

$$
x \in\|K((\varphi \wedge \psi) \rightarrow\langle K\rangle((\varphi \wedge \psi) \wedge K((\varphi \wedge \psi) \rightarrow \theta)))\| .
$$

\section{References}

1. Abramsky, S. (1991). Domain theory in logical form. Annals of Pure and Applied Logic, 51(1), 1-77.

2. Aiello, M., Pratt-Hartmann, I., van Benthem, J. (2007). Handbook of spatial logics. Germany: Springer Verlag.

3. Alchourrón, C.E., Gärdenfors, P., Makinson, D. (1985). On the logic of theory change: partial meet contraction and revision functions. Journal of Symbolic Logic, 50(2), 510-530.

4. Baltag, A., Bezhanishvili, N., Özgün, A., Smets, S. (2013). The topology of belief, belief revision and defeasible knowledge. In Logic, rationality, and interaction - 4th international workshop, LORI 2013, Hangzhou, China, October 9-12, 2013, Proceedings (pp. 27-40).

5. Baltag, A., Bezhanishvili, N., Özgün, A., Smets, S. (2014). The topology of full and weak belief. In Proceedings of the 11th international Tbilisi symposium on logic, language, and computation (TbiLLC 2015) revised selected papers (pp. 205-228).

6. Baltag, A., Bezhanishvili, N., Özgún, A., Smets, S. (2016). Justified belief and the topology of evidence. In Proceedings of the 23rd workshop on logic, language, information and computation (WOLLIC 2016) (pp. 83-103).

7. Baltag, A., \& Smets, S. (2006). Conditional doxastic models: a qualitative approach to dynamic belief revision. Electronic Notes in Theoretical Computer Science, 165, 5-21. Proceedings of the 13th Workshop on Logic, Language, Information and Computation (WoLLIC 2006).

8. Baltag, A., \& Smets, S. (2008). A qualitative theory of dynamic interactive belief revision. Texts in Logic and Games, 3, 9-58.

9. Barwise, J. (1988). Three views of common knowledge. In Proceedings of the 2nd conference on theoretical aspects of reasoning about knolwedge (pp. 365-379).

10. Baskent, C. (2007). Topics in subset space logic. Master's thesis, ILLC, University of Amsterdam.

11. Baskent, C. (2011). Geometric public announcement logics. In Murray, R.C. \& McCarthy, P.M. (Eds.), Preceedings of the 24th Florida artificial intelligence research society conference (FLAIRS-24) (pp. 87-88).

12. Battigalli, P., \& Siniscalchi, M. (2002). Strong belief and forward induction reasoning. Journal of Economic Theory, 105, 356-391.

13. Beklemishev, L., \& Gabelaia, D. (2014). Topological interpretations of provability logic (pp. 257290). Netherlands: Springer.

14. Bezhanishvili, G., Bezhanishvili, N., Lucero-Bryan, J., van Mill, J. (2015). S4.3 and hereditarily extremally disconnected spaces. Georgian Mathemetical Journals, 22, 469-475.

15. Bezhanishvili, G., Esakia, L., Gabelaia, D. (2005). Some results on modal axiomatization and definability for topological spaces. Studia Logica, 81(3), 325-355.

16. Bezhanishvili, G., Esakia, L., Gabelaia, D. (2011). Spectral and $T_{0}$-spaces in d-semantics. In Logic, language, and computation - 8th international Tbilisi symposium on logic, language, and computation, LNAI. Revised selected papers (Vol. 6618, pp. 16-29).

17. Bezhanishvili, N., \& van der Hoek, W. (2014). Structures for epistemic logic. In Baltag, A., \& Smets, S. (Eds.) Johan van Benthem on logic and information dynamics (pp. 339-380) Springer International Publishing.

18. Bjorndahl, A., \& Özgün, A. (2017). Logic and topology for knowledge, knowability, and belief. In Proceedings of 16th conference on theoretical aspects of rationality and knowledgle (TARK 2017), vol. 251 of electronic proceedings in theoretical computer science (pp. 88-101).

19. Blackburn, P., de Rijke, M., Venema, Y. (2001). Modal logic, vol 53 of cambridge tracts in theoretical computer scie. Cambridge: Cambridge University Press.

20. Blaszczyk, A., Rajagopalan, M., Szymanski, A. (1993). Spaces which are hereditarily extremely disconnected. Journal of Ramanujan Mathematical Society, 8(1-2), 81-94.

21. Boutilier, C. (1990). Conditional logics of normality as modal systems. In Shrobe, H.E., Dietterich, T.G. \& Swartout, W.R. (Eds.), AAAI (pp. 594-599). 
22. Chagrov, A.V., \& Zakharyaschev, M. (1997). Modal logic, vol. 35 of oxford logic guides. Oxford University Press.

23. Dabrowski, A., Moss, L.S., Parikh, R. (1996). Topological reasoning and the logic of knowledge. Annals of Pure and Applied Logic, 78(1), 73-110.

24. DeRose, K. (2009). The case for contextualism. 1st Edn. New York: Oxford University Press.

25. Dugundji, J. (1965). Topology, Allyn and Bacon series in advanced mathematics. Prentice Hall.

26. Engelking, R. (1989). General topology, 2nd edn. Vol. 6. Berlin: Heldermann Verlag.

27. Esakia, L. (2001). Weak transitivity-restitution. Study in Logic, 8, 244-254. In Russian.

28. Esakia, L. (2004). Intuitionistic logic and modality via topology. Annals of Pure and Applied Logic, 127(1), 155-170.

29. Gabelaia, D. (2001). Modal definability in topology. Master's thesis, ILLC, University of Amsterdam.

30. Georgatos, K. (1993). Modal logics for topological spaces. Ph.D. thesis, City University of New York.

31. Georgatos, K. (1994). Knowledge theoretic properties of topological spaces. In Proceedings of knowledge representation and reasoning under uncertainty: logic at work (pp. 147-159).

32. Gettier, E. (1963). Is justified true belief knowledge? Analysis, 23, 121-123.

33. Ichikawa, J.J., \& Steup, M. (2013). The analysis of knowledge. In Zalta, E.N. (Ed.) The Stanford encyclopedia of philosophy. Metaphysics Research Lab, Stanford University, fall 2013 edition.

34. Kelly, K. (1996). The logic of reliable inquiry. Oxford University Press.

35. Klein, P. (1971). A proposed definition of propositional knowledge. Journal of Philosophy, 68, 471482 .

36. Kraus, S., Lehmann, D., Magidor, M. (1990). Nonmonotonic reasoning, preferential models and cumulative logics. Artificial Intelligence, 44(1-2), 167-207.

37. Kudinov, A., \& Shehtman, V. (2014). Derivational modal logics with the difference modality (pp. 291334). Netherlands: Springer.

38. Lamarre, P., \& Shoham, Y. (1994). Knowledge, certainty, belief, and conditionalisation (abbreviated version). In Doyle, J., Sandewall, E., Torasso, P. (Eds.) Principles of knowledge representation and reasoning (pp. 415-424). The Morgan Kaufmann Series in Representation and Reasoning. Morgan Kaufmann.

39. Lehrer, K., \& Paxson, T.J. (1969). Knowledge: undefeated justified true belief. Journal of Philosophy, 66, 225-237.

40. Lenzen, W. (1978). Recent work in epistemic logic Vol. 30. North Holland: Acta Philosophica Fennica.

41. McKinsey, J.C.C. (1941). A solution of the decision problem for the Lewis systems S2 and S4, with an application to topology. The Journal of Symbolic Logic, 6(4), 117-134.

42. McKinsey, J.C.C., \& Tarski, A. (1944). The algebra of topology. Annals of Mathematics (2), 45, 141-191.

43. Moss, L.S., \& Parikh, R. (1992). Topological reasoning and the logic of knowledge. In Proceedings of 4th conference on theoretical aspects of computer science (TARK 1992) (pp. 95-105). Morgan Kaufmann.

44. Noiri, T. (1988). Charaterizations of extremally disconnected spaces. Indian Journal of Pure and Applied Mathematics, 19, 325-329.

45. Nozick, R. (1981). Philosophical explanations Cambridge. MA: Harvard University Press.

46. Özgün, A. (2013). Topological models for belief and belief revision. Master's thesis, ILLC, University of Amsterdam.

47. Özgủn, A. (2017). Evidence in epistemic logic: a topological perspective. Ph.D. thesis, LORIA. Université de Lorraine and ILLC, University of Amsterdam.

48. Parikh, R., Moss, L.S., Steinsvold, C. (2007). Topology and epistemic logic. In Handbook of spatial logics (pp. 299-341). Springer Verlag.

49. Rott, H. (2004). Stability, strength and sensitivity: converting belief into knowledge. Erkenntnis, 61(23), 469-493.

50. Sikorski, R. (1964). Boolean algebras. Berlin-Heidelberg-Newyork: Springer-Verlag.

51. Sosa, E. (1999). How to defeat opposition to Moore. Nos, 33, 141-153.

52. Stalnaker, R. (1968). A theory of conditionals. In Studies in logical theory (Vol. 2, pp. 98-112). Oxford: Blackwell.

53. Stalnaker, R. (2006). On logics of knowledge and belief. Philosophical Studies, 128(1), 169-199.

54. Steinsvold, C. (2007). Topological models of belief logics. Ph.D. thesis, City University of New York.

55. Tarski, A. (1938). Der Aussagenkalkül und die Topologie. Fundamenta Mathematicae, 31, $103-134$. 
56. Troelstra, A.S., \& van Dalen, D. (1988). Constructivism in mathematics: an introduction., vol. 1 and 2 of studies in logic and the foundations of mathematics. Amsterdam.

57. Tsao-Chen, T. (1938). Algebraic postulates and a geometric interpretation for the Lewis calculus of strict implication. Bulletin of the American Mathematical Society, 44(10), 737-744.

58. van Benthem, J. (2004). Dynamic logic for belief revision. Journal of Applied Non-Classical Logics, 14, 2004.

59. van Benthem, J. (2011). Logical dynamics of information and interaction. New York: Cambridge University Press.

60. van Benthem, J., \& Bezhanishvili, G. (2007). Modal logics of space. In Handbook of spatial logics (pp. 217-298). Springer.

61. van Benthem, J., Bezhanishvilli, G., ten Cate, B., Sarenac, D. (2005). Modal logics for products of topologies. Studia Logica, 84(3), 369-392.

62. van Benthem, J., \& Pacuit, E. (2011). Dynamic logics of evidence-based beliefs. Studia Logica, 99(1), 61-92.

63. van Benthem, J., \& Sarenac, D. (2005). The geometry of knowledge. Aspects of Universal Logic, 17.

64. van Ditmarsch, H., van der Hoek, W., Kooi, B. (2007). Dynamic epistemic logic, 1st Edn. Springer Publishing Company, Incorporated.

65. van Fraassen, B. (1995). Fine-grained opinion, probability, and the logic of full belief. Journal of Philosophical logic, 24, 349-377.

66. Vickers, S. (1989). Topology via logic, vol 5 of cambridge tracts in theoretical computer science. Cambridge: Cambridge University Press.

67. Voorbraak, F. (1993). As far as I know. Ph.D. thesis, Utrecht University.

68. Wang, Y., \& Cao, Q. (2013). On axiomatizations of public announcement logic. Synthese, 190, 103134.

69. Williamson, T. (2000). Knowledge and its limits. Oxford Univ. Press.

70. Wolter, F. (1993). Lattices of modal logics. Ph.D. thesis, Free University Berlin.

71. Zvesper, J. (2010). Playing with information. Ph.D thesis, ILLC, University of Amsterdam. 